\author{
Federal Reserve Bank of New York \\ Staff Reports
}

\title{
The Inflation-Output Trade-Off Revisited
}

\author{
Gauti B. Eggertsson \\ Marc P. Giannoni
}

Staff Report No. 608

March 2013

Revised May 2013

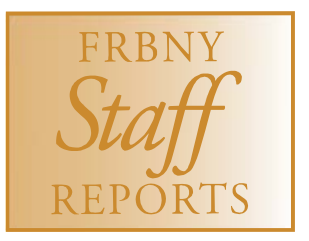

This paper presents preliminary findings and is being distributed to economists and other interested readers solely to stimulate discussion and elicit comments. The views expressed in this paper are those of the authors and are not necessarily reflective of views at the Federal Reserve Bank of New York or the Federal Reserve System. Any errors or omissions are the responsibility of the authors. 


\title{
The Inflation-Output Trade-Off Revisited
}

Gauti B. Eggertsson and Marc P. Giannoni

Federal Reserve Bank of New York Staff Reports, no. 608

March 2013; revised May 2013

JEL classification: E00, E13, E40, E58

\begin{abstract}
A rich literature from the 1970s shows that as inflation expectations become more and more ingrained, monetary policy loses its stimulative effect. In the extreme, with perfectly anticipated inflation, there is no trade-off between inflation and output. A recent literature on the interest-rate zero lower bound, however, suggests there may be some benefits from anticipated inflation when the economy is in a liquidity trap. In this paper, we reconcile these two views by showing that while it is true, at positive interest rates, that inflation loses its stimulative effects as it becomes better anticipated, the opposite holds true at the zero bound. Indeed, at the zero bound, the more accurately the public anticipates inflation, the greater is the expansionary effect of inflation on output. This leads us to revisit the trade-off between inflation and output and to show how radically it changes in the face of demand shocks large enough to bring the economy into a liquidity trap. Instead of vanishing once inflation becomes anticipated, the trade-off between inflation and output increases substantially and may become arbitrarily large. In such cases, raising the inflation target in a liquidity trap can be very stimulative.
\end{abstract}

Key words: zero lower bound, trade-off

Eggertsson: Brown University (e-mail: gauti_eggertsson@brown.edu). Giannoni: Federal Reserve Bank of New York and Center for Economic Policy Research (e-mail:

marc.giannoni@ny.frb.org). This paper is based on a plenary talk given at the conference "Zero Bound on Interest Rates and New Directions in Monetary Policy,” Wilfrid Laurier University, Waterloo, Ontario, October 2011. The views expressed in this paper are those of the authors and do not necessarily reflect the position of the Federal Reserve Bank of New York or the Federal Reserve System. 


\section{Introduction}

"What we know, or should know, from the past is that once inflation becomes anticipated and ingrained - as it eventually would - then the stimulating effects are lost." (Paul Volcker, former Chairman of the Federal Reserve, NY Times, 9/19/11)

Once the central bank lowers the nominal interest rate down to zero, it cannot provide more stimulus via nominal interest rate cuts. It can however make monetary policy more expansionary by increasing inflation expectations, as a recent literature has suggested. This mechanism works in virtually all modern macroeconomic models because aggregate demand depends not on the level of the nominal interest rate but instead on the real interest rate - the difference between the nominal rate and expected inflation. Hence even if the real rate cannot be lowered by nominal interest rate cuts at the zero bound, it can still be reduced through increases in inflation expectations, thus making spending today more attractive relative to the future. This is, for example, highlighted in Krugman (1998) and Eggertsson and Woodford (2003), but a large literature finds similar conclusions in various settings.

Given this relatively broad consensus in the modern literature, it may seem somewhat surprising that even though several advanced economies found themselves with interest rates close to zero following the crisis of 2008, and unemployment at levels not seen in decades, no major central bank has deliberately attempted to raise inflation (with the possible very recent exception of the Bank of Japan). ${ }^{1}$ This contrasts sharply with the response of various policy makers during the Great Depression. At that time, several economies left the gold standard and some deliberately allowed inflation to rise (see e.g. Eggertsson (2008) for discussion of the US; Eichengreen and Sachs (1985) present evidence that countries that exited the gold standard and allowed inflation to rise experienced a much more rapid recovery than those that did not.)

What is the reason for this disconnect? The most obvious reason is summarized by Paul Volcker, former Chairman of the Federal Reserve, in an editorial in the New York Times (excerpted above). In it, he argued forcefully against any increase in the inflation target during the recession following the crisis of 2008 . Why? The recovery from the Great Depression is not the only example of increasing inflation. Indeed, it is well documented that inflation also rose in many countries during the 1970s. And while the countries which left the gold standard early experienced a more robust recovery from the Great Depression, it is challenging to make that case for the countries that experienced excessive inflation in the 1970s. If anything, it seems that those countries that allowed inflation to rise achieved just high inflation with little benefit in terms of employment. Moreover, once inflation had risen in 1970s, its high level arguably became somewhat of a hindrance rather than a help for growth. ${ }^{2}$ Indeed, while Paul Volcker managed to bring down inflation from double digits to relatively low rates in the early 1980s, he did so by generating a well-documented

\footnotetext{
${ }^{1}$ It is interesting to note that in the US, the Federal Open Market Committee (FOMC) announced following its December 2012 meeting that it intends to maintain short-term interest rates close to zero "at least as long as the unemployment rate remains above 6-1/2 percent, inflation between one and two years ahead is projected to be no more than a half percentage point above the Committee's 2 percent longer-run goal, and longer-term inflation expectations continue to be well anchored." So while the FOMC does not explicitly seek to raise inflation above its long-run target, it is willing to tolerate an inflation rate temporarily above its long-run target provided that the unemployment rate remains high. As we will see, the theoretical analysis presented here is strongly supportive of a strategy of that kind.

${ }^{2}$ John Cochrane (2012), for example, argues, "it's a rare Phillips curve in which raising expected inflation is a good thing. It just gives you more inflation, with if anything less output and employment."
} 
recession (Goodfriend and King (2005)) often referred to as the "Volcker recession." The most obvious reason for the reluctance of policy makers to embrace positive inflation during the crisis of 2008 is thus related to the widely perceived lesson from the 1970s, that once anticipated, inflation is at best neutral and at worst a hindrance for further recovery. ${ }^{3}$

The purpose of this paper is to reconcile the lessons of the 1970s and 1980s with the more recent literature which argues that higher inflation is useful to increase demand. Many early rational expectations models emphasized that monetary policy has real effects because people do not correctly anticipate the future when setting their prices (hence the preset prices differ from their market clearing levels). Similar assumptions are also hard-wired in most modern New Keynesian models. It would thus seem that once one accepts this proposition, anticipated inflation should provide few beneficial effects since money will be largely neutral once expectations have adjusted, at least at some horizon. What we will illustrate, however, is that monetary neutrality - as given for example by a New Classical expectations-augmented Phillips curve (Lucas (1972), Kydland and Prescott (1977), Sargent and Wallace (1975), Barro and Gordon (1983)) - is not a legitimate argument against increasing inflation expectations as long as the zero bound is binding. In fact, as long as the zero bound is binding and an equilibrium exists, we will see that even with the New Classical Phillips curve which embeds a very strong neutrality result, the benefit of inflation becomes bigger rather than smaller as inflation becomes more and more anticipated. This result turns Volcker's argument on its head at the zero bound. It is the main result of the paper.

The basic reason for this result is relatively subtle and relies upon the fact that once the New Classical framework is amended to incorporate aggregate demand, full neutrality of monetary policy no longer applies, at least once the zero bound is binding. The notion that monetary policy is neutral when inflation is perfectly anticipated is simply inconsistent with the demand side of the model if the short-term interest rate is constrained by the zero lower bound. The two sides of the model - aggregate demand and aggregate supply — clash so that no equilibrium exists. How should this non-existence result be interpreted? We suggest an interpretation and a simple extension that generates existence in a natural way and leads us to revisit the trade-off between inflation and output. This in turn strengthens the case for inflation in large demand slumps.

In what follows, we start our analysis in Section 2 by revisiting the classic explanation for the coincident high inflation and high unemployment in the 1970s via a standard New Classical Phillips curve of the form

$$
\pi_{t}=\kappa \hat{Y}_{t}+E_{t-1} \pi_{t}
$$

where $\pi_{t}$ is inflation $\hat{Y}_{t}$ is output in deviation from steady state (or alternatively a measure of an "output gap") and $E_{t-1} \pi_{t}$ is the expectation of inflation at time $t$ formed at a time when either some prices or wages were set (we will provide explicit microfoundations for this relationship assuming that some prices are fixed one period in advance, in Section 3.1). If inflation is fully anticipated, then $\pi_{t}=E_{t-1} \pi_{t}$ and this equation says that $\hat{Y}_{t}=0$. Hence, any positive inflation, once anticipated, would seem to loose its expansionary effect, as suggested by Volcker in the opening quote of the paper.

We point out in Section 3 that this particular result is obtained by looking exclusively at the "supply equation" of the model (1). We then introduce a "demand" side, which makes clear that this result is

\footnotetext{
${ }^{3}$ In broad terms, the absence of trade-offs between inflation and output is the main lesson of the rational expectation revolution of the 1970s and 1980s which arguably resulted in several Nobel prices, e.g. as exemplified by the work by Phelps (1967), Lucas (1972), Sargent and Wallace (1975) and Kydland and Prescott (1977) cited by the Nobel committee.
} 
only partial and potentially misleading in general equilibrium. The demand side is standard: Consumers choose a stream of consumption over the infinite horizon and policy can affect demand via variation in the nominal interest rate, so that inflation and output are determined in equilibrium. A key result is that if shocks are large enough for the zero bound on the short-term nominal interest rate to bind then, given expectations, output is demand determined. In that case, and with a reasonable specification of policy, output demanded is always below "potential," i.e. $\hat{Y}_{t}<0$, according to aggregate demand. Moreover, even if inflation is perfectly anticipated, we show that the solution $\hat{Y}_{t}=0$ implied by the supply side is inconsistent with the demand-side equilibrium conditions of the model. Hence a key result of this paper is a non-existence result: The proposed solution of equation (1) suggested above, and implicitly assumed in Volcker's commentary - that if inflation is anticipated then $\hat{Y}_{t}=0$ - leads to non-existence of an equilibrium in the model. In other words, there is a clash between the demand and the supply side of the model.

How can this clash be resolved? What is the interpretation of the non-existence result? We propose one interpretation of the non-existence result in Section 4 by relaxing the assumption of perfect foresight while still maintaining the assumption of rational expectations. We show that when introducing a little uncertainty in the model, an equilibrium exists but it leads to very "bad" outcomes: In this equilibrium the "clash of the two equations" is resolved in favor of the demand side, and the solution features an output collapse and deflation. Once inflation becomes perfectly anticipated, output and inflation collapse completely, which ultimately yields non-existence of the equilibrium. We refer to this situation as a contractionary black hole. Interestingly we show that in this equilibrium - as long as it exists - anticipated inflation is far from neutral. The benefits of anticipated inflation, in fact, become extremely large and approach infinity as the model converges towards the contractionary black hole. This turns the Volcker argument on its head: Rather than finding that inflation becomes less effective for stimulating output when it becomes more and more anticipated, the reverse is true at the zero bound. This result is closely related to the "flexibility paradox" illustrated for example in Eggertsson (2011), Christiano Eichenbaum and Rebelo (2011), Werning (2012) and Bhattarai, Eggertsson and Schoenle (2012). These papers show that the economy suffers a worse drop in output in a liquidity trap once prices become more flexible, conditional on a given shock. This is paradoxical since if prices were fully flexible, then output would not drop at all according to the supply side of the model. Similarly, here, we see that as inflation becomes more anticipated then higher inflation becomes more stimulative. But paradoxically, if inflation were fully anticipated then the supply relationship (equation (1)) would seem to suggest that inflation should have no effect at all.

Section 5 analyzes the short-run equilibrium and shows that when the equilibrium exists in the medium and long run, a sufficiently long-lasting adverse shock may result in a collapse in prices and output in the short run. Section 6 revisits the inflation output trade-off in light of these results. It establishes an important distinction between traditional static trade-offs between inflation and output in the same period, and intertemporal trade-offs between output in a period and inflation in a later period. Section 7 reviews results from earlier sections in the context of a non-linear version of the model, thus guaranteeing that our findings are not due to the use of a linearized version of the model, which we exploit in parts of the paper for tractability (and easier comparison with the earlier literature that uses that framework).

We picked the New Classical model as an initial benchmark since it offers the sharpest example of neutralities when inflation is anticipated, and is often used in popular discussions describing the 1970s or 
to argue that attempting to increase inflation would be futile in current circumstances. In that model, the "short-run" lasts only one period. Section 8 considers the same issue in the standard New Keynesian model now popular in the literature. In that model, the short-run is no longer just defined by just one period. As a result, the non-existence issue also arises but in terms of a constraint on how long the slump can last without implying an explosion of key variables in the model. In the New Keynesian model, we will also find that as inflation becomes more anticipated, it becomes more expansionary at the zero bound. This leads us once again to revisit the trade-off between inflation and output, and to point out how it radically changes at the zero bound.

Before proceeding, we should make clear two separate points. First, our non-existence result depends importantly on the monetary policy commitment assumed. Our assumed policy implies that the central bank would never allow inflation to increase above its implicit inflation target. Since the zero bound imposes no restriction on raising the nominal interest rate (and thus on curbing too high inflation) this implies that in equilibrium the inflation rate will always be at or below (when the zero bound is binding) the central bank's implicit inflation target. This kind of equilibrium is implied by both optimal policy under discretion (Eggertsson (2006)) and a standard Taylor rule (Eggertsson and Woodford (2003)). With different types of policy commitments, for example if the central bank followed a price level target or its fully optimal policy, the problem of non-existence of the equilibrium may not arise. Indeed, a commitment to a price level target would imply that any deflation at the zero bound will eventually be undone with future inflation, once the zero bound is no longer binding. This highlights the importance of a policy rule that accommodates some short-term expected inflation at the zero bound, which is consistent with our main point.

Second, the benefits of inflation that we highlight here stem from increasing inflation in the medium run, i.e., in a relatively short period after the shock which brought the economy to the zero bound has subsided. Hence if the shock does not last very long, there is no need for a permanent rise in the long-term inflation target. The results presented here suggest that it may be beneficial to let the near-term inflation target move over time in a way that depends on the state of the economy, when the zero bound constrains the economy. ${ }^{4}$

\section{The Phelps problem}

Before presenting a more detailed microfounded model, it is useful to remind the reader of what Sargent (1999) refers to as the "Phelps problem," a problem made classic in the seminal papers of Kydland and Prescott (1977) and Barro and Gordon (1983). Consider a government that minimizes

$$
\min _{\pi_{t}} E_{0} \sum_{t=0}^{\infty} \beta^{t}\left\{\pi_{t}^{2}+\lambda\left(\hat{Y}_{t}-\hat{Y}^{*}\right)^{2}\right\}
$$

subject to

$$
\pi_{t}=\kappa \hat{Y}_{t}+E_{t-1} \pi_{t}
$$

\footnotetext{
${ }^{4}$ Of course, it may be desirable to let near-term inflation deviate from the long-run target even if the zero lower bound is not binding, when other kinds of frictions exist (see, e.g., Giannoni and Woodford (2005) for an analysis of optimal monetary policy in models incorporating various frictions).
} 


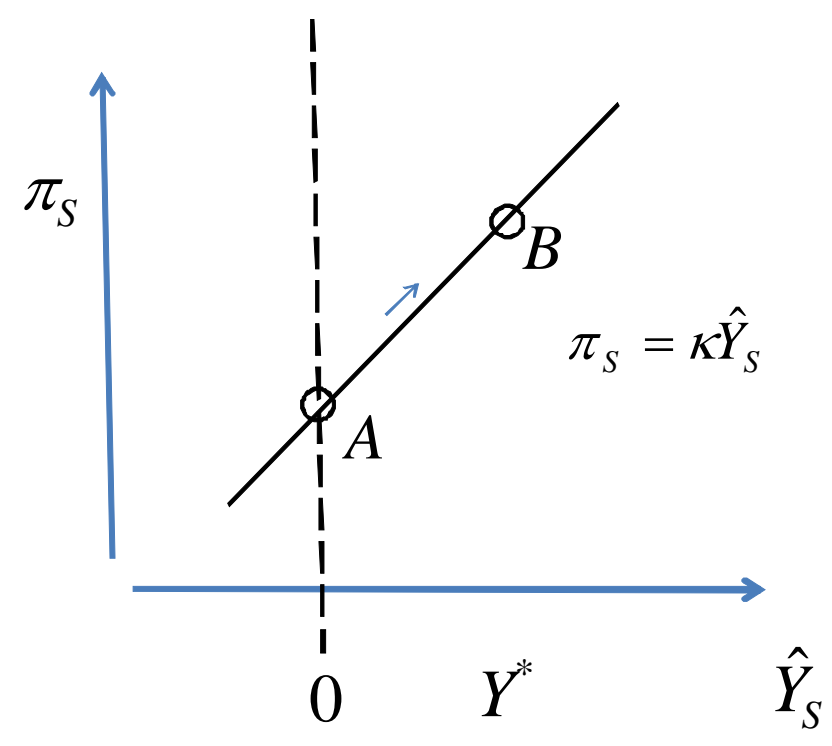

Figure 1: The tradeoff between inflation and output when inflation expectations are fixed.

where $\pi_{t}$ is inflation, $\hat{Y}_{t}$ is output in percent deviation from steady state, $\hat{Y}^{*}$ is the first-best level of output in percent deviation from steady state, and $E_{t-1} \pi_{t}$ is the expectation of inflation at time $t$ formed at time $t-1$. As we shall see, the Phillips curve restriction (3) emerges naturally as a approximation to our microfounded model in Section 3.1. ${ }^{5}$ Let us define the short run as a period $t=S$ in which inflation expectations have not adjusted, but are instead at zero so that $E_{S-1} \pi_{S}=0$. Let us define a medium run as the period in which expectations have adjusted so that $\pi_{M}=E_{M-1} \pi_{M}=E_{S} \pi_{M}$. For now, there is no difference between long and medium run but we will make a sharper distinction between these shortly.

Figure 1 shows the output and interest rates in the model in the short run, given by the schedule $\pi_{S}=\kappa \hat{Y}_{S}$. We see that in the short run the government can achieve higher output by creating (unexpected) inflation, and the trade-off between inflation and output is given by $\kappa^{-1}$, i.e. a one percentage point increase in inflation increases output by $\kappa^{-1}$ percent. Hence in the short run the government has a menu of choices of inflation output pairs on the solid line dotted by $A$ and $B$. For large enough inflation, the government can even achieve the first best output $\hat{Y}^{*}$ that minimizes the objective (2).

The key point of the literature from the 1970's is that this menu of choices is an illusion once expectations adjust. In particular, once inflation becomes fully anticipated, $\pi_{t}=E_{t-1} \pi_{t}$ and the AS equation (3) becomes $\pi_{M}=\kappa \hat{Y}_{M}+\pi_{M}$ which implies that $\hat{Y}_{M}=0$. Once the policy is anticipated the government can only choose between different inflation rates on dashed curve from $A$ to $C$ in Figure 2 without any improvement in output. In particular, suppose the private sector anticipated that the government would

\footnotetext{
${ }^{5}$ Depending on the types of exogenous shocks considered, the variable $\hat{Y}_{t}$ may alternatively be interpreted as capturing an "output gap" expressed in deviations from steady state.
} 


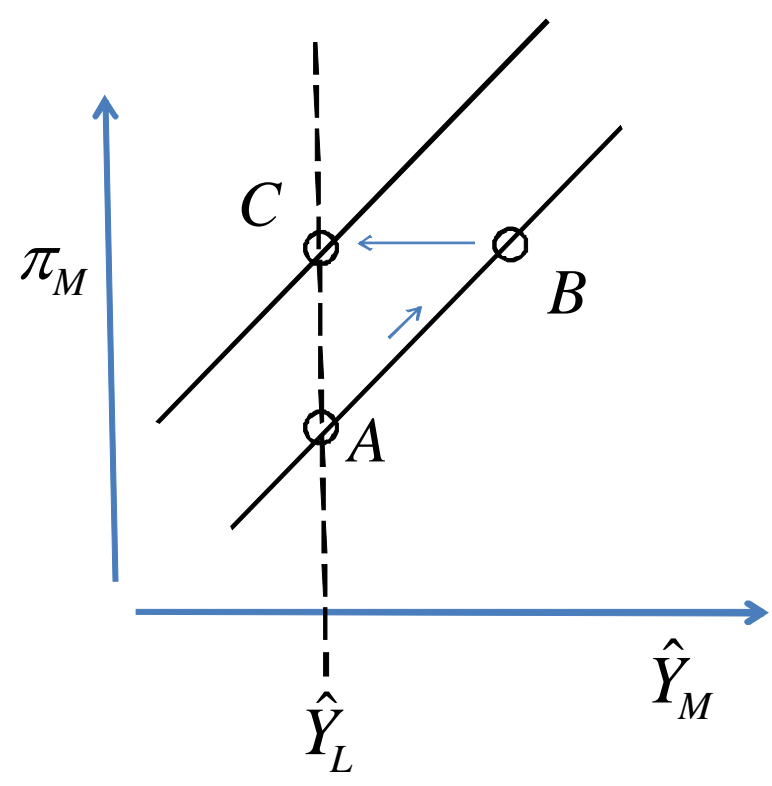

Figure 2: If inflation expectations adjust fully they eliminate any output gains from inflation.

try to achieve point B (where output is at potential). In this case $E_{S} \pi_{M}=\pi_{M}$ and the AS curve shifts as shown in the figure so that once the government chooses $\pi_{M}$ there are no gains in output, only excessive inflation. This is at core the Volcker's remark "What we know, or should know, from the past is that once inflation becomes anticipated and ingrained - as it eventually would - then the stimulating effects are lost."

\section{Introducing demand into the Phelps problem: The problem of non-existence}

In the last section we reviewed a classic example of how an expectations-augmented Phillips curve suggest that there is no medium run trade-off between inflation and output, where the medium run is defined by the fact that expectations have adjusted. Missing in this picture, however, is a demand side. How does the government "select" an inflation rate? Does the modeling of how this is accomplished change the basic picture? In this section we extend the model to explicitly take account of the aggregate demand side, which then leads us to explore how the equilibrium is achieved. The key point is that once the demand side is introduced, then the benign solution described above, derived from the supply side with $\hat{Y}_{t}=0$ (at any anticipated rate of inflation) can no longer be achieved under certain conditions. It clashes directly with the demand side of the model. Resolving the clash between the demand and supply sides of this model leads to some interesting findings. 


\subsection{Microfoundations}

This subsection summarizes the microfoundations of the model. The impatient or experienced reader can move directly to the next section (the microfoundations spelled out are largely covered, e.g., in Woodford (2003)). There is a continuum of households of measure 1. The representative household maximizes utility

$$
E_{t} \sum_{T=t}^{\infty} \beta^{T-t} \xi_{T}\left[u\left(C_{T}\right)-v\left(l_{T}\right)\right]
$$

where $\beta \in(0,1)$ is a discount factor, $C_{t}$ is a Dixit-Stiglitz aggregate of consumption of each of a continuum of differentiated goods, $C_{t} \equiv\left[\int_{0}^{1} c_{t}(j)^{\frac{\theta-1}{\theta}} d j\right]^{\frac{\theta}{\theta-1}}$ with an elasticity of substitution equal to $\theta>1, P_{t}$ is the Dixit-Stiglitz price index, $P_{t} \equiv\left[\int_{0}^{1} p_{t}(j)^{1-\theta} d j\right]^{\frac{1}{1-\theta}}$, and $l_{t}$ is an aggregate measuring the quantity of labor supplied. The disturbance $\xi_{t}$ is a preference shock, and $u($.$) is an increasing concave function while v($.$) is$ an increasing convex function. The period budget constraint can be written as

$$
P_{t} C_{t}+B_{t}=\left(1+i_{t-1}\right) B_{t-1}+\int_{0}^{1} Z_{t}(j) d j+P_{t} W_{t} l_{t}-T_{t}
$$

where $B_{t}$ denotes the quantity of one-period riskless bonds, $i_{t}$ is the nominal interest rate, $Z_{t}(i)$ corresponds to profits that are distributed lump sum to the households, and $W_{t}$ is the real wage rate. For the budget constraint to be well defined, and Ponzi schemes not possible, we require that ${ }^{6}$

$$
\left(1+i_{t}\right) B_{t}>-\sum_{T=t+1}^{\infty} E_{t+1}\left[Q_{t+1, T}\left(\int_{0}^{1} Z_{T}(j) d j+P_{T} W_{T} l_{T}-T_{T}\right)\right]
$$

and

$$
\sum_{T=t}^{\infty} E_{t}\left[Q_{t, T}\left(\int_{0}^{1} Z_{T}(j) d j+P_{T} W_{T} l_{T}-T_{T}\right)\right]<\infty
$$

where $Q_{t, T} \equiv \prod_{s=t+1}^{T} Q_{s-1, s}$ denotes the stochastic discount factor.

Households take prices and wages as given and maximize utility subject to the budget constraint. This gives rise to the first-order conditions for the optimal consumption allocation

$$
u_{c}\left(C_{t}\right) \xi_{t}=\left(1+i_{t}\right) \beta E_{t}\left[u_{c}\left(C_{t+1}\right) \xi_{t+1} \Pi_{t+1}^{-1}\right]
$$

where we define $\Pi_{t} \equiv P_{t} / P_{t-1}$ and we note that $\left(1+i_{t}\right)^{-1}=E_{t} Q_{t, t+1}$, and the condition for the optimal labor decision

$$
W_{t}=\frac{v_{l}\left(l_{t}\right)}{u_{c}\left(C_{t}\right)} .
$$

Furthermore we impose the constraint that the nominal interest rate has to be positive ${ }^{7}$

$$
i_{t} \geq 0
$$

There is a continuum of firms of measure 1. A fraction $\gamma$ of the firms sets prices freely every period while a fraction $1-\gamma$ sets prices one period in advance. Each firm sets its price and then hires the labor inputs

\footnotetext{
${ }^{6}$ See Woodford (2003, Chap 2.) for discussion.

${ }^{7}$ This constraint can be interpreted as a consequence of the household maximization problem if there exists money in the economy as a nominal store of value.
} 
necessary to meet any demand that may be realized taking wages as given. A unit of labor produces one unit of output. The preferences of households imply a demand for good $i$ of the form $y_{t}(j)=Y_{t}\left(\frac{p_{t}(j)}{P_{t}}\right)^{-\theta}$, where $Y_{t}=C_{t}$ is aggregate output. We assume that all profits are paid out as dividends and that the firm seeks to maximize profits. Profits can be written as $Z_{t}(j)=p_{t}(j) Y_{t}\left(p_{t}(j) / P_{t}\right)^{-\theta}-W_{t} P_{t} Y_{t}\left(p_{t}(j) / P_{t}\right)^{-\theta}$, where $j$ indexes the firm. The first-order condition for the firms that set their prices freely every period is given by

$$
\frac{p_{t}(1)}{P_{t}}=\frac{\theta}{\theta-1} W_{t}
$$

while the first-order condition of the firms that set their prices one period in advance is

$$
E_{t-1}\left[u_{c}\left(Y_{t}\right) Y_{t}\left(\frac{p_{t}(2)}{P_{t}}\right)^{-\theta}\left(\frac{p_{t}(2)}{P_{t}}-\frac{\theta}{\theta-1} W_{t}\right)\right]=0,
$$

where we have used

$$
Y_{t}=l_{t}=C_{t}
$$

The aggregate price level then implies

$$
1=\gamma \frac{p_{t}(1)}{P_{t}}+(1-\gamma) \frac{p_{t}(2)}{P_{t}}
$$

After using (13) to substitute for $C_{t}$ and $l_{t}$ in the above equations, we can define an equilibrium as a set of stochastic processes $\left\{\frac{p_{t}(1)}{P_{t}}, \frac{p_{t}(2)}{P_{t}}, \Pi_{t}, Y_{t}, W_{t}, i_{t}\right\}$ that satisfy equations (8)-(12), (14) for a given shock process $\left\{\xi_{t}\right\}$ and for given fiscal and monetary policies that satisfy equations (6)-(7).

\subsection{A Linear Quadratic Approximation}

The model just outlined can be approximated via log-linear-quadratic approximation around a deterministic steady state. We use this form of the model for simplicity and to connect it with the earlier literature. As we will show, however, nothing is lost with this simplified exposition; the same basic insights carry through to the nonlinear version of the model discussed in Section 7.

A log-linear approximation of the firm's optimal pricing decisions given by equations (11)-(14) delivers the aggregate supply equation (3) where $\kappa>0$ depends on some of the underlying parameters of the model. In addition, a log-linear approximation of the demand side of the model ((8) using (13)) yields

$$
\hat{Y}_{t}=E_{t} \hat{Y}_{t+1}-\sigma\left(i_{t}-E_{t} \pi_{t+1}-r_{t}^{e}\right)
$$

where $\hat{Y}_{t} \equiv \log \left(Y_{t} / \bar{Y}\right), \pi_{t} \equiv \log \left(P_{t} / P_{t-1}\right), i_{t}$ now refers to $\log \left(1+i_{t}\right)$ in terms of our previous notation, and $\sigma>0$. The "natural" rate of interest $r_{t}^{e} \equiv \bar{r}+E_{t} \log \left(\xi_{t} / \xi_{t+1}\right)$ is an exogenous variable that depends on $\bar{r} \equiv \log \beta^{-1}>0$ and the preference shock $\xi_{t}$, and that reflects the desirability of consuming in the present relative to the future. The interest rate bound can once again be expressed as

$$
i_{t} \geq 0 \text {. }
$$

Reviewing the "Phelps problem" characterized above, it should be clear why previous authors have often abstracted from the demand side. Since the nominal interest rate does not directly appear in the objective (2) or in equation (3), there is no loss of generality in assuming that instead of choosing the nominal interest rate, $i_{t}$, the government chooses directly either $\pi_{t}$ or $\hat{Y}_{t}$ that satisfy the restriction (3). 
The nominal interest rate consistent with these levels of inflation and output can then just be backed out of (15). ${ }^{8}$ However, nothing in this way of proceeding guarantees that the implied interest rate must be nonnegative. Therefore, when one explicitly accounts for the zero bound, one need to incorporate aggregate demand in the analysis.

Introducing aggregate demand however requires a more complete specification of monetary policy. As mentioned in the introduction, we do not focus here on the characterization of the optimal (commitment) policy. ${ }^{9}$ Instead, we focus on the implications of a policy that commits to bring inflation to its target level whenever possible, that is, as long as the zero bound is not binding. A simple way to formulate such a policy is to model the interest-rate setting according to the simple rule:

$$
i_{t}=\max \left\{0, r_{t}^{e}+\pi_{t}^{*}+\phi_{\pi}\left(\pi_{t}-\pi_{t}^{*}\right)\right\}
$$

where $\pi_{t}^{*}$ is the inflation target of the government and where we assume that $\phi_{\pi}>1$ so that the so-called "Taylor principle" applies, whereby the nominal interest rate is raised more than one-for-one with inflation around the inflation target. ${ }^{10}$ If inflation is perfectly anticipated, then $\pi_{t}=\pi_{t}^{*}=E_{t-1} \pi_{t}$. Moreover, if the zero bound is not binding then (16) will implement the inflation target in every period (this result is special to the fact that $r_{t}^{e}$ is the only shock in the model).

In the case considered in Section 2, as inflation becomes anticipated, the equilibrium moves from point $B$ to $C$ : Higher inflation leads to no output gains once fully anticipated. We see from equation (15) that the net effect of this is simply an increase in the nominal interest rate, i.e., $i_{S}=\pi_{M}$. This is arguably in broad terms - what happened during the "great inflation" of the 1970s: as inflation expectations rose, nominal rates rose as well, while output remained depressed. It would seem, then, at least when studying the 1970s, that the demand side is only relevant to "back out" the nominal interest rate implied by the equilibrium.

However, when the zero bound becomes binding, the demand side of the model affects the equilibrium as well. Once the nominal interest rate drops to zero, equation (15) is no longer just a pricing equation for the nominal interest rate. Instead, it starts playing a role in determining the overall number good demanded which will have critical effects on the equilibrium determination. This is the case that we now turn to.

\subsection{Short, medium, long run and non-existence}

We now consider the case in which the zero bound can be binding, and explore how that changes the basic picture. To do so we assume that there is an unexpected negative shock $r_{t}^{e}<\bar{r}$ in period zero that we call the "short run." The short run is defined by the fact that at that time expectations have not adjusted in the model; they remain at "steady state" so that $E_{-1} \pi_{S}=\pi_{L}^{*}$. We then assume that the shock stays at its negative level in the next period (which we call the medium run) and then reverts back to normal in the third period which we call the "long run." Expectations have fully adjusted in the medium and the long

\footnotetext{
${ }^{8} \mathrm{~A}$ similar argument can also be made for the money supply. We can add money in the utility function into our framework, so that the government's choice of the nominal interest rate is then modeled via its choice of the money supply. We omit this detail here.

${ }^{9}$ Such an analysis can be found, e.g., in Eggertsson and Woodford (2003).

${ }^{10} \mathrm{We}$ could alternatively write the policy rule as $i_{t}=\max \left\{0, r_{t}^{e}+\pi_{t}+\phi_{\pi}\left(\pi_{t}-\pi_{t}^{*}\right)\right\}$. All the results presented below remain valid with appropriate modifications to some of the parameters. Note that in this case, the Taylor principle would require $\phi_{\pi}>0$.
} 


\begin{tabular}{|c|c|c|}
\hline $\begin{array}{c}\text { Short run } \\
\mathbf{S}\end{array}$ & $\begin{array}{c}\text { Medium run } \\
\mathbf{M}\end{array}$ & $\begin{array}{l}\text { Long run } \\
\qquad\end{array}$ \\
\hline $\begin{array}{l}\text { Shocks } r_{S}^{e}<\bar{r} \\
\text { - expectations } \\
\text { NOT adjusted }\end{array}$ & $\begin{array}{l}\text { Shocks: } r_{M}^{e}=r^{e}{ }_{S} \\
\text { - expectations } \\
\text { fully adjusted. } \\
\text { Perfect foresight }\end{array}$ & $\begin{array}{l}\text { No shocks: } r_{L}=\bar{r} \\
\text { - expectations adjusted } \\
\text { Key assumption: } \\
\text { Zero lower bound is not } \\
\text { binding in long-run }\end{array}$ \\
\hline
\end{tabular}

Figure 3: Time protocol according to Assumption 1.

run so that the only difference between the medium and the long run is the absence of the shock in the long run. Similarly the only difference between the medium and the short run is that expectations have adjusted in the medium run, but not in the short run. To summarize:

A1 Consider the three periods $t=S, M, L$. In period $t=S$ there is an unexpected shock $r_{t}^{e}=r_{S}^{e}<\bar{r}$. In period $t=M$ the shock is still $r_{t}^{e}=r_{S}^{e}$. In periods $t \geq L$ the shock is back at steady state $r_{t}^{e}=r_{L}=\bar{r}$. While the shock is unexpected in period $t=S$, so that $E_{S-1} \pi_{S}=\pi^{*}$, there is perfect foresight between $S, M$, and $L$. Periods $t>L$ are identical to $L$ : there are no shocks and agents have perfect foresight.

Let us start with some preliminaries, namely proving that in the long run, $t \geq L$, with the policy rule (16), there is a unique bounded solution in which $\pi_{t}=\pi_{L}^{*}=\pi^{*}$. For simplicity, we assume that the zero bound is not binding in the long run and thus we exclude the possibility of self-fulfilling liquidity traps which is a subject of another branch of the literature. ${ }^{11}$ We relax this restriction in Section 7 below and show that our main results are not affected by it.

Proposition 1 Suppose A1, that $\bar{r}>-\pi_{L}^{*}, \phi_{\pi}>1$ and that the nominal interest rate is always positive in the long run $t \geq L$. Then the model (3), (15)-16) implies a unique bounded long-run equilibrium $\left\{\pi_{L}, \hat{Y}_{L}, i_{L}, E_{L} \pi_{L+1}, E_{L} \hat{Y}_{L+1}\right\}$ given by $\pi_{L}=E_{L} \pi_{L+1}=\pi_{L}^{*}, i_{L}=\bar{r}+\pi_{L}^{*}$ and $\hat{Y}_{L}=E_{L} \hat{Y}_{L+1}=0$.

Proof. Given Assumption A1, perfect foresight between periods $M$ and $L$ implies that $E_{L-1} \pi_{L}=$ $E_{M} \pi_{L}=\pi_{L}$. It follows from equation (3) that for all $t \geq L$

$$
\pi_{t}=\kappa \hat{Y}_{t}+\pi_{t}
$$

or simply that that $\hat{Y}_{t}=0$ for $t \geq L$. This implies that for any $t \geq L(15)$ simplifies to

$$
i_{t}=E_{t} \pi_{t+1}+\bar{r}
$$

It follows from (16) and (17) that

$$
E_{t} \pi_{t+1}+\bar{r}=\bar{r}+\pi_{L}^{*}+\phi_{\pi}\left(\pi_{t}-\pi_{L}^{*}\right)
$$

\footnotetext{
${ }^{11}$ Eggertsson and Woodford (2003), for instance, suggest some policies which can explude these type of equilibria.
} 


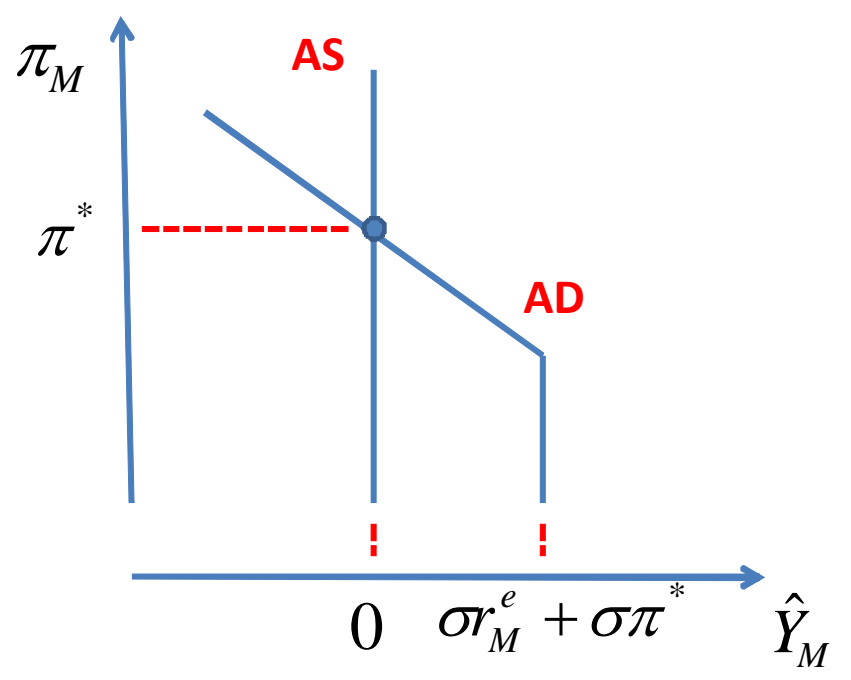

Figure 4: Equilibrium at positive interest rate.

or equivalently that

$$
\pi_{t}=\phi_{\pi}^{-1} E_{t} \pi_{t+1}+\left(1-\phi_{\pi}^{-1}\right) \pi_{L}^{*}
$$

Iterating this forward yields

$$
\pi_{t}=\phi_{\pi}^{-j} E_{t} \pi_{t+j}+\left(1-\phi_{\pi}^{-1}\right) \pi_{L}^{*} \sum_{s=1}^{j} \phi_{\pi}^{-(s-1)}=\pi_{L}^{*}
$$

for any bounded $\left\{\pi_{t}\right\}_{t \geq L}$ since $\phi_{\pi}>1$ and $\lim _{j \rightarrow \infty} \phi_{\pi}^{-j} E_{t} \pi_{t+j}=0$.

Our focus, next, is on the equilibrium determination in the medium run given our specification for policy, before we move to the short-run. Consider the aggregate supply (AS) equation (3) in the medium run. It is given by a vertical line

$$
\hat{Y}_{M}=\kappa^{-1}\left(\pi_{M}-E_{S} \pi_{M}\right)=\kappa^{-1}\left(\pi_{M}-\pi_{M}\right)=0
$$

where the last equation follows from the fact that we assume perfect foresight between the short and medium run in A1.

The aggregate demand (AD) equation, obtained by combining (16) with (15), yields

$$
\hat{Y}_{M}=\left\{\begin{array}{cc}
-\sigma \phi_{\pi}\left(\pi_{M}-\pi_{M}^{*}\right)+\sigma\left(\pi_{L}^{*}-\pi_{M}^{*}\right) & \text { if } r_{M}^{e}+\pi_{M}^{*}+\phi_{\pi}\left(\pi_{M}-\pi_{M}^{*}\right)>0 \\
\sigma r_{M}^{e}+\sigma \pi_{L}^{*} & \text { if } r_{M}^{e}+\pi_{M}^{*}+\phi_{\pi}\left(\pi_{M}-\pi_{M}^{*}\right) \leq 0
\end{array}\right.
$$

The two curves are plotted up in Figure 4 for a realization of the shock $r_{M}^{e}$ satisfying $r_{M}^{e}+\pi_{M}^{*}+$ $\phi_{\pi}\left(\pi_{M}-\pi_{M}^{*}\right)>0$. Since inflation is perfectly anticipated in the medium run, we see that that the AS 


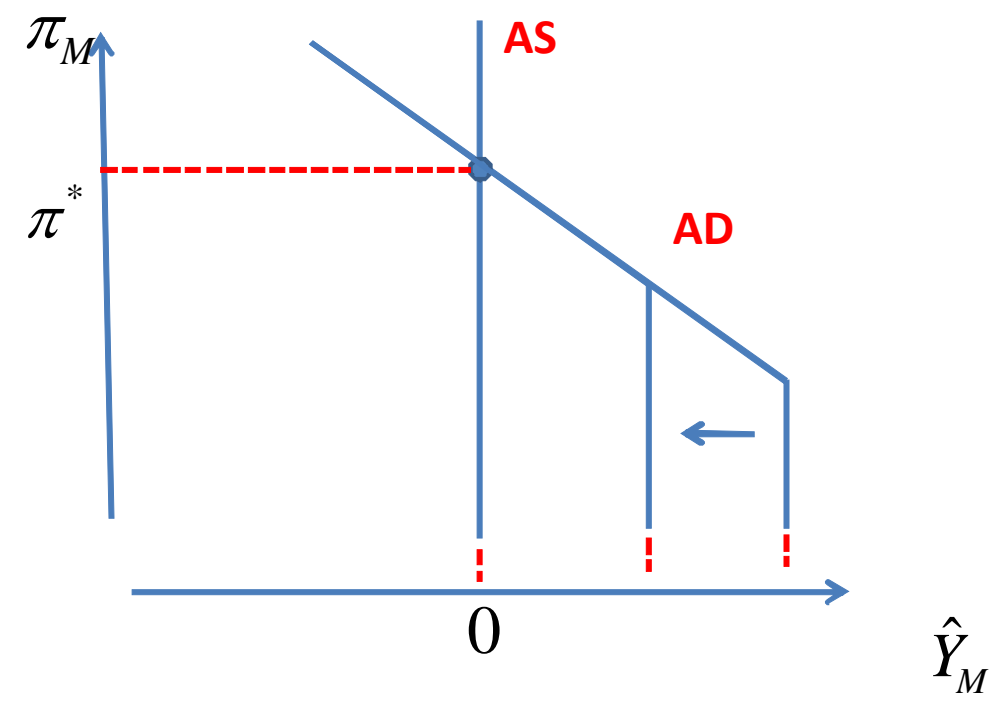

Figure 5: A shock to $r_{S}^{e}$ has no effect on output or inflation as long as the zero bound is not binding.

curve is vertical. Meanwhile, the AD curve is downward sloping in inflation. This is due to the fact that $\phi_{\pi}>1$ so that the central bank will reduce the nominal interest rate more than one-to-one with a fall in inflation (and vice versa), thus stimulating spending as inflation drops as shown in the first row of (18). Yet, there is a limit to how much the central bank can stimulate spending by cutting the nominal interest rate. If medium term inflation, $\pi_{M}$, is low enough so that the zero bound is binding then $\hat{Y}_{M}$ is given by the second row of (18), i.e. $\hat{Y}_{M}=\sigma r_{M}^{e}+\sigma \pi_{L}^{*}$. In this case the output demanded does not depend upon realized inflation (the ratio of prices today relative to yesterday). Instead demand only depends upon expected inflation, $E_{M} \pi_{L}\left(=\pi_{L}^{*}\right)$, which determines the price of goods tomorrow relative to the price today. In the previous case, this relative price was also affected through realized inflation because inflation affected the nominal interest rate setting of the central bank; this is no longer the case once the interest rate is pinned at zero. An equilibrium is determined by the intersection of the $\mathrm{AS}$ and $\mathrm{AD}$ equation. At positive interest rate we see that this equilibrium determination happens at $\pi_{M}=\pi_{M}^{*}$ and $\hat{Y}_{M}=0$.

Figure 5 shows the effect of the shock $r_{M}^{e}$ being more negative. This shifts the AD curve leftward. If this shock is small enough, then the only thing that happens is that the nominal interest rate is reduced but inflation stays at $\pi_{M}^{*}$ and output at potential.

There is nothing in the model, however, that prevents the AD curve from shifting even further than in Figure 5. In particular consider the following shock: $r_{M}^{e}<-\pi_{M}^{*}$. This size of the shock shifts the AD curve to the left of the AS curve. Clearly the two curves do not intersect. In other words there is no equilibrium. To summarize:

Proposition 2 Suppose A1, $r_{M}^{e}<-\pi_{L}^{*}, \bar{r}>-\pi_{L}^{*}, \phi_{\pi}>1$, and that the nominal interest rate is always 


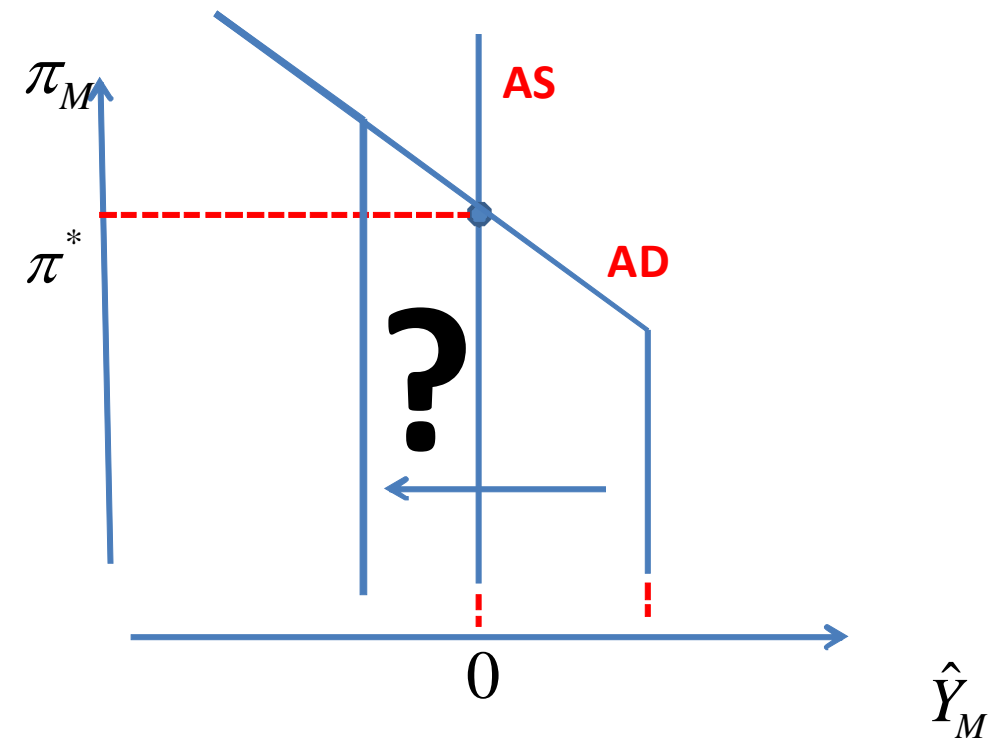

Figure 6: Aggregate demand and aggregate supply clash: No equilibrium.

positive in the long run. Then there exists no bounded equilibrium in the medium run that satisfies equations (3) and (15)-(16).

Proof. See Appendix.

How should this proposition, that no solution exist, be interpreted? That is the issue we now turn to.

\section{Generating existence: Demand wins}

The aggregate supply equation and the aggregate demand equation in Figure 5 are pointing in two different directions. On the one hand the aggregate supply equation simply imposes that $\hat{Y}_{M}=0$. If everybody perfectly anticipates the future, then prices act as if they are perfectly flexible and output is thus at potential. Meanwhile, the aggregate demand equation imposes that demand must be below its steady state, i.e., $\hat{Y}_{M}<0$. For households to be willing to buy all supplied goods, the real interest rate must be sufficiently negative, but this is not possible if expected inflation is below $\pi_{t}^{*}$ without a negative nominal interest rate. Evidently demand and supply clash - no level of output and inflation satisfy both equations at the same time. What is particularly noteworthy here is that some of the policy discussion reviewed in the introduction - and professional consensus - seems to be driven by an intuition which is derived exactly when this clash occurs but by only using the AS equation.

At heart of the issue is the strong neutrality imposed by the AS equation in combination with a temporary shock to the $\mathrm{AD}$ equation that reduces demand. Once inflation is anticipated, then output cannot deviate from potential according to the AS equation, thus hard-wiring no trade-off between inflation 


\section{Short run Medium run Long run}

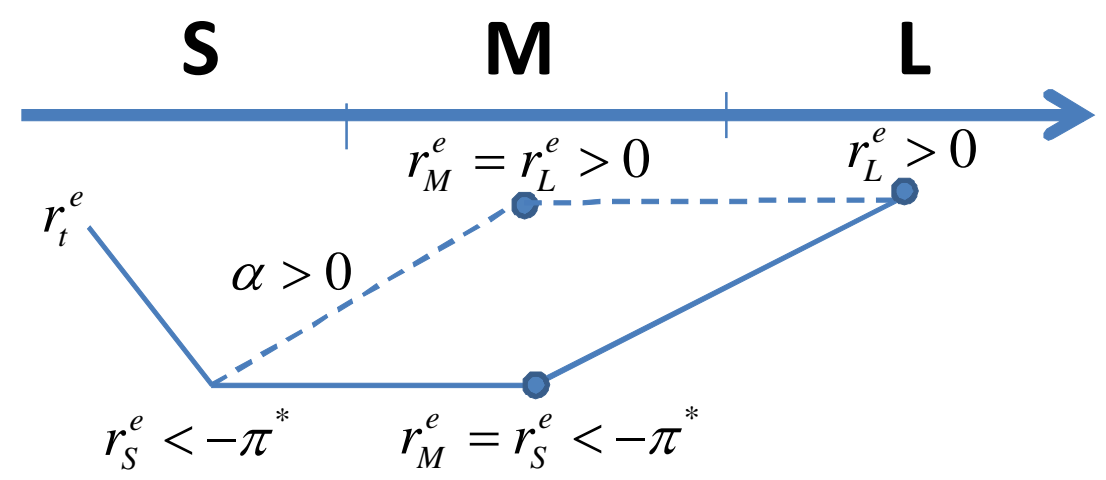

Figure 7: Introducing uncertainty. With probability $\alpha$, the natural rate $r_{M}^{e}$ in the medium run reaches its long run value $r_{L}^{e}>0$. With probability $1-\alpha$ it remains at $r_{S}^{e}<-\pi^{*}$.

and output. This excludes - by assumption - the possibility of a long protracted demand driven slump, a situation in which those firms with preset prices produce less than the others. Given that we have some prices set one period in advance (so output is demand determined in equilibrium) a critical property of the model is perfect foresight by the agents, i.e. no uncertainty and rational expectations. Here we will deviate from this assumption in a way that seems quite reasonable — while still maintaining rational expectations — and show how the equilibrium is determined. This, we argue, gives some insight into the interpretation of the non-existence result.

Recall our previous assumption that there is a shock in the short-run that stays "on" in the medium run and finally reverts back to steady state in the long run. Let us now deviate from perfect foresight by assuming that there is a probability, $\alpha$, that the shock reverts back to steady state in the medium run rather than in the long run. This seemingly minor extension generates existence in the model. Moreover, it clarifies how the model should be interpreted which has important policy implications.

A2 Consider three periods $t=S, M, L$. In period $t=S$ there is an unexpected shock $r_{t}^{e}=r_{S}^{e}<\bar{r}$ In period $t=M$ the shock is still $r_{t}^{e}=r_{S}^{e}$ with probability $(1-\alpha)$ and $r_{t}^{e}=r_{L}^{e}>0$ with probability $\alpha$. In periods $t \geq L$ the shock is back at steady state $r_{t}^{e}=r_{L}^{e}=\bar{r}$. The shock is unexpected in period $t=S$ but people form rational expectations about the shock in period $t=M$ using the correct probability distribution of the model $(\alpha)$.

The long run is as before $\hat{Y}_{L}=0, \pi_{L}=\pi_{L}^{*}$ and $i_{L}=r_{L}^{e}+\pi_{L}^{*}$. Moving to the medium run, we now have two possible states, i) that the shock reverts back to steady state $r_{M}^{e}=r_{L}^{e}>0$ (which we call "high") or ii) that the shock remains at $r_{M}^{e}=r_{S}^{e}<-\pi_{M}^{*}$, in which case the nominal interest rate is constrained to be at zero. The model then solves the following six equations in the medium run

$$
\pi_{M}^{j}=\kappa \hat{Y}_{M}^{j}+\alpha \pi_{M}^{h i g h}+(1-\alpha) \pi_{M}^{l o w}, \text { for } j=\text { low or high }
$$




$$
\begin{aligned}
\hat{Y}_{M}^{\text {low }} & =\hat{Y}_{L}-\sigma\left(i_{M}^{\text {low }}-\pi_{L}^{*}-r_{S}^{e}\right) \\
\hat{Y}_{M}^{h i g h} & =\hat{Y}_{L}-\sigma\left(i_{M}^{\text {high }}-\pi_{L}^{*}-r_{L}^{e}\right) \\
i_{M}^{\text {low }}=0 \quad \text { and } \quad & i_{M}^{h i g h}=r_{L}^{e}+\pi_{M}^{*}+\phi_{\pi}\left(\pi_{M}^{h i g h}-\pi_{M}^{*}\right)
\end{aligned}
$$

while in the short run it solves

$$
\begin{gathered}
\hat{Y}_{S}=\alpha \hat{Y}_{M}^{h i g h}+(1-\alpha) \hat{Y}_{M}^{l o w}+\sigma \alpha \pi_{M}^{h i g h}+\sigma(1-\alpha) \pi_{M}^{l o w}+\sigma r_{S}^{e} \\
\pi_{S}=\kappa \hat{Y}_{S} .
\end{gathered}
$$

The model's equilibrium is described in the following proposition:

Proposition 3 Suppose A2, that $r_{S}^{e}<-\pi_{M}^{*}, \bar{r}>-\pi_{L}^{*}, \phi_{\pi}>1$ and that the nominal interest rate is always positive in the long run $t \geq L$. Then with $\alpha>0$ there exists a unique bounded solution to (19)-(24) given by

$$
\begin{aligned}
\hat{Y}_{M}^{\text {high }} & =-\frac{1-\alpha}{\alpha} \sigma\left(r_{S}^{e}+\pi_{L}^{*}\right) \\
\pi_{M}^{\text {high }} & =\frac{1-\alpha}{\phi_{\pi}^{\alpha}} r_{S}^{e}+\frac{1}{\alpha \phi_{\pi}} \pi_{L}^{*}+\frac{\phi_{\pi}-1}{\phi_{\pi}} \pi_{M}^{*} \\
\hat{Y}_{M}^{\text {low }} & =\sigma\left(r_{S}^{e}+\pi_{L}^{*}\right) \\
\pi_{M}^{\text {low }} & =\frac{1-\alpha+\phi_{\pi} \kappa \sigma}{\phi_{\pi} \alpha} r_{S}^{e}+\frac{1+\phi_{\pi} \kappa \sigma}{\phi_{\pi} \alpha} \pi_{L}^{*}+\frac{\phi_{\pi}-1}{\phi_{\pi}} \pi_{M}^{*} \\
\hat{Y}_{S} & =\sigma\left(\frac{1-\alpha}{\alpha} \frac{1+\phi_{\pi} \kappa \sigma}{\phi_{\pi}}+1\right) r_{S}^{e}+\sigma \frac{(1-\alpha) \phi_{\pi} \kappa \sigma+1}{\phi_{\pi} \alpha} \pi_{L}^{*}+\sigma \frac{\phi_{\pi}-1}{\phi_{\pi}} \pi_{M}^{*} \\
\pi_{S} & =\kappa \hat{Y}_{S} .
\end{aligned}
$$

Proof. The solution is obtained by solving (19)-(24) (see Appendix for details).

The model equilibrium exists in the medium run, now that there is no longer perfect foresight. For instance, if the shock is in the low state, then the AS equation is given by

$$
\pi_{M}^{l o w}=\kappa \hat{Y}_{M}^{l o w}+\alpha \pi_{M}^{h i g h}+(1-\alpha) \pi_{M}^{l o w}
$$

or

$$
\pi_{M}^{l o w}=\frac{\kappa}{\alpha} \hat{Y}_{M}^{l o w}+\pi_{M}^{\text {high }}
$$

We can now use our solution for $\pi_{M}^{\text {high }}$ to show that inflation and output are related in the medium run according to

$$
\pi_{M}^{l o w}=\frac{1-\alpha+\phi_{\pi} \kappa \sigma}{\phi_{\pi} \alpha} \sigma^{-1} \hat{Y}_{M}^{l o w}+\phi_{\pi}^{-1} \pi_{L}^{*}+\left(1-\phi_{\pi}^{-1}\right) \pi_{M}^{*}
$$

Equation (25) shows that now the AS curve is now no longer vertical in the medium run in the low state, once we take into account the equilibrium determination in the "high state" using the equilibrium conditions from last proposition. Instead, it is upward sloping in the inflation output space as shown in Figure 8. This figure shows the AS and AD curves conditional on the shock remaining at its short-run 


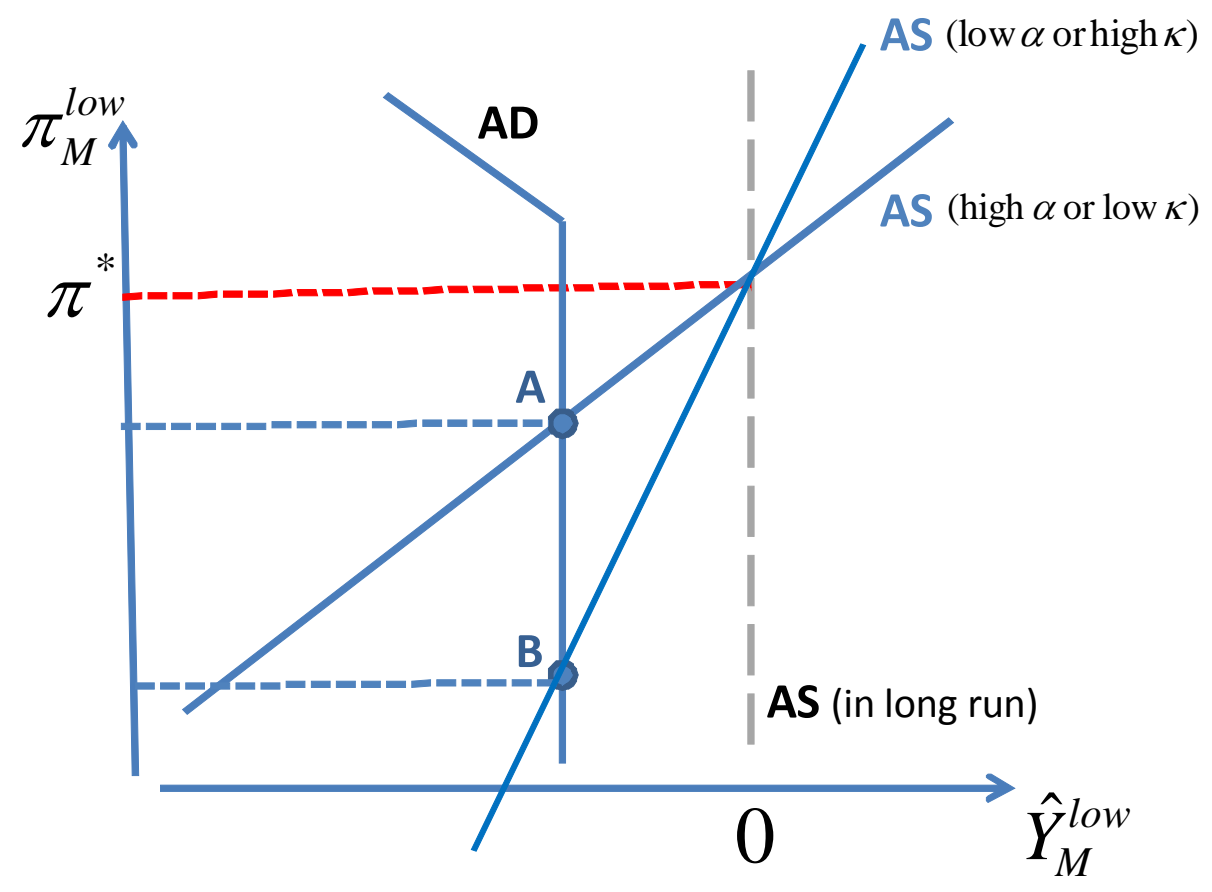

Figure 8: The clash between aggregate demand and aggregate supply resolved: Demand wins.

value, i.e. $r_{M}^{e}=r_{S}^{e}$, hence the value of the shock is the same as in our previous exercise when no solution existed. What generates existence here is the fact that inflation is no longer perfectly anticipated, as there is more than one state of the world in the medium run and there is a probability $\alpha$ to reach the "high" state where the zero bound is no longer binding. As long as there is some uncertainty about outcomes, actual inflation is no longer equal to its anticipated level and the AS curve is upward sloping in the outputinflation space as shown in Figure 8. The reason is simple. In the microfounded model, the firms which have their prices fixed in advance are committed to supply whatever is demanded by the consumers. As people start demanding fewer goods and services, for some prices given, less labor is hired, which depresses wages. Lower wages reduce in turn the firms' marginal costs, so that the firms that can freely adjust their prices choose to lower them. It follows that the fewer output is bought from firms, the greater the price deflation. This basic logic is only broken in the knife-edge case when all prices are perfectly anticipated. In that case demand does not have any bite, since the economy acts "as if" prices are perfectly flexible.

Plotting the downward sloping AD curve and the upward sloping AS curve in Figure 8 reveals an equilibrium in point A. As people reduce their demand for goods and services, AD shifts left, reducing output, while the AS curve determines the degree of deflation associated with that level of aggregate demand. In other words, in the clash between demand and supply in the medium run, the aggregate demand wins. 


\section{Uncertainty and contractionary black holes in the short run}

While output is pinned down by aggregate demand in the medium run, the amount of deflation "needed" to clear the market depends on the AS curve. Thus deflation in the medium run - conditional on the shock being in the "low state" so that the zero bound is still binding — plays no role for medium-run output, as the latter is already pegged down by the vertical AD curve. As we have seen in Figure 8 the degree of deflation needed to clear the market in the medium run depends on the slope of the AS equation. This slope depends on $\alpha$. In particular as the probability people assigned to being in the "low" state in the medium run goes up (i.e. $\alpha \rightarrow 0$ ) the deflation needed increases (see point B in Figure 8). Eventually the AS curve becomes vertical - the model converges to perfect foresight - and no solution exists.

How much deflation is needed? That depends on the coefficient $\frac{1-\alpha+\phi_{\pi} \kappa \sigma}{\phi_{\pi} \alpha}$. As we have already indicated, this slope becomes steeper with lower values of $\alpha$. Some uncertainty will therefore in general lead to a "static" trade-off between inflation and output (more on this in the next section). Beyond that, an important determinant of this slope is the coefficient $\kappa$. Let us just emphasize two key components of this coefficient which we decompose as $\kappa \equiv \frac{\gamma}{1-\gamma} \psi^{-1}$. First, we see that $\kappa$ depends on the overall level of nominal rigidities - which is here indexed by the fraction of firms that set their prices one period in advance $1-\gamma$. If prices are very "sticky" then the AS curve is very flat, hence a small decline in inflation below target inflation is needed to equilibrate the market. Second, the degree of real rigidities $\psi$. This coefficient measures the strategic complementarities in the model, i.e., the extent to which firms tend to cut their own prices in response to declines in other firms' prices. To the extent that these rigidities are high and the price adjustment is asynchronous, firms that face a drop in demand may not lower their prices much even if they can, as other firms are constrained to maintain their prices unchanged. In such a case, even a large drop in output will generate only a modest decline in the price level. These points are worth making in the context of the recent world economic crisis, where many countries have seen downward drops in inflation pressures, yet those have been relatively contained. This can be viewed as evidence in favor of high degree of nominal and/or real rigidities in the context of this model. ${ }^{12}$

While the amount of deflation in the medium run has no effect on output in the medium run, it has important consequences for output in the short run. In particular, we will now see that the higher the degree of deflation, the bigger the drop in output in the short-run. What is interesting here is to note that what will in general trigger a great drop in inflation in the medium run are things one would typically associate with a stabilizing force, namely anything that makes the AS curve more vertical, be it real or nominal rigidity or a movement towards inflation becoming more anticipated (i.e. $\alpha$ is closer to 0 ). But now we will obtain precisely the opposite conclusion, higher real or nominal rigidities, or more anticipated inflation/deflation is destabilizing (this is reminiscent of the result documented in Eggertsson (2012), Christiano, Eichenbaum and Rebelo (2011) and Werning (2012)). Provided $\alpha>0$, the solution in the short run is now well defined. Consider the solution in the short-run, taking the medium term solution derived in the last section as given. Given $\pi_{M}$ and $\hat{Y}_{M}$, the solution is given by solving together (23) and (24), i.e.,

$$
\hat{Y}_{S}=E_{S} \hat{Y}_{M}+\sigma E_{S} \pi_{M}+\sigma r_{S}^{e}
$$

\footnotetext{
${ }^{12}$ As noted by Eggertsson and Krugman (2012), another reason for relatively modest drop in inflation is that the underlying shock that triggers the zero bound leads to a simultaneous "cost push" shock. They show how a debt deleveraging shock can have this effect.
} 


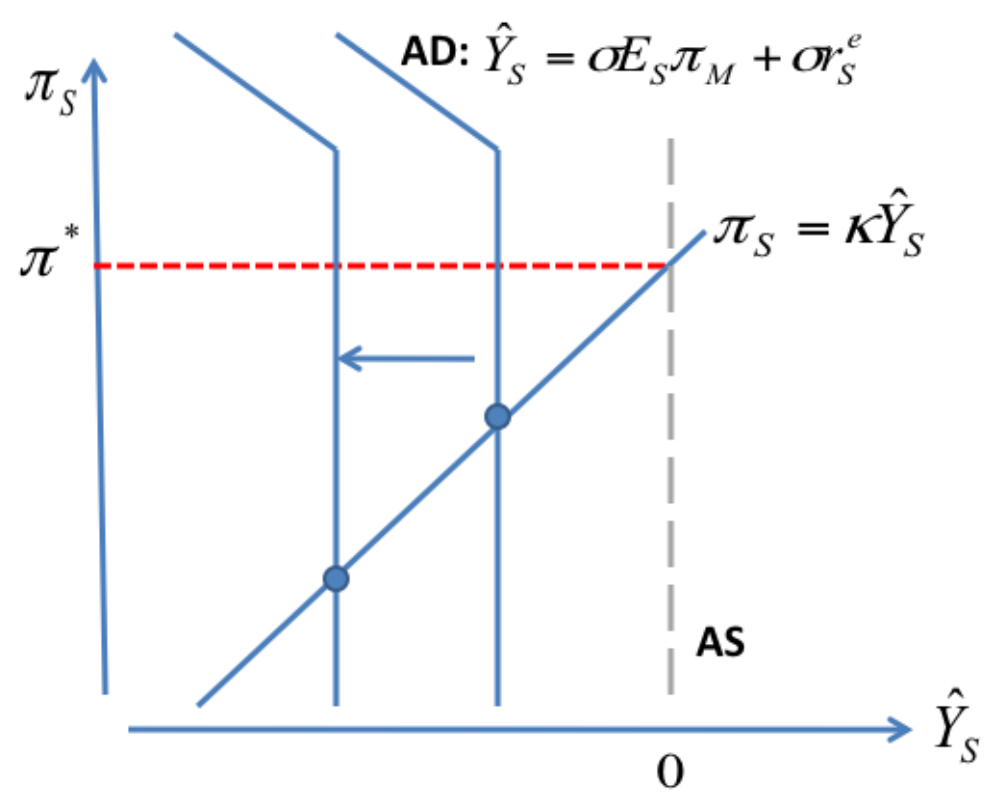

Figure 9: Deflation in the medium run reduces short run output since expected deflation increases the real interest rate and thus contracts demand.

$$
\pi_{S}=\kappa \hat{Y}_{S}
$$

which are plotted in Figure 9.

We see that this figure is essentially the same as Figure 8. There is one important new element, however, lurking in the background. The AD curve will now shift not only due to the shock $r_{S}^{e}$. It will also shift due to expected deflation - given by $E_{S} \pi_{M}$ - which is no longer only given by the exogenous inflation target $\pi_{M}^{*}$. Instead, expected inflation/deflation is a function of the endogenous deflation in medium run which we saw in Figure 8. It can be expressed as

$$
E_{S} \pi_{M}=\frac{(1-\alpha)\left(\kappa \sigma \phi_{\pi}+1\right)}{\alpha \phi_{\pi}} r_{S}^{e}+\frac{\phi_{\pi}-1}{\phi_{\pi}} \pi_{M}^{*}+\frac{(1-\alpha) \kappa \sigma \phi_{\pi}+1}{\alpha \phi_{\pi}} \pi_{L}^{*} .
$$

This illustrates that the backward shift in aggregate demand is now amplified by the expected deflation in the medium run, resulting in further backward shift in aggregate demand as shown in Figure 9. Most importantly, perhaps, medium term inflation expectation are declining in the probability $\alpha$. That is, the more likely the "low" state $1-\alpha$ then the higher the deflation in the low state, and thus the larger the expected deflation in the short run, as captured by the first term in (26). The expected deflation increases without a bound, i.e., as $\alpha \rightarrow 0$ then $E_{S} \pi_{M} \rightarrow-\infty$ leading to an output collapse $\left(\hat{Y}_{S} \rightarrow-\infty\right)$. Note that an increase in $\kappa$ works in the same direction (e.g. due to higher real or nominal flexibility). This is what we refer to as a contractionary black hole. To summarize

Proposition 4 Assume A2, $r_{S}^{e}+\pi_{L}^{*}<0$ and $\phi_{\pi}>1$. As the probability of the medium-run recession increases, i.e., $\alpha \rightarrow 0$, the recession in the short run increases without a bound $\hat{Y}_{S} \rightarrow-\infty$.

Proof. The expression for $\hat{Y}_{S}$ in proposition 3 can be rewritten as $\hat{Y}_{S}=\sigma\left(\frac{(1-\alpha) \phi_{\pi} \kappa \sigma+1}{\phi_{\pi} \alpha}\right)\left(r_{S}^{e}+\pi_{L}^{*}\right)+$ $\sigma\left(1-\phi_{\pi}^{-1}\right)\left(r_{S}^{e}+\pi_{M}^{*}\right)$. Since $r_{S}^{e}+\pi_{L}^{*}<0$ by assumption, $\lim _{\alpha \rightarrow 0} \hat{Y}_{S}=-\infty$. 


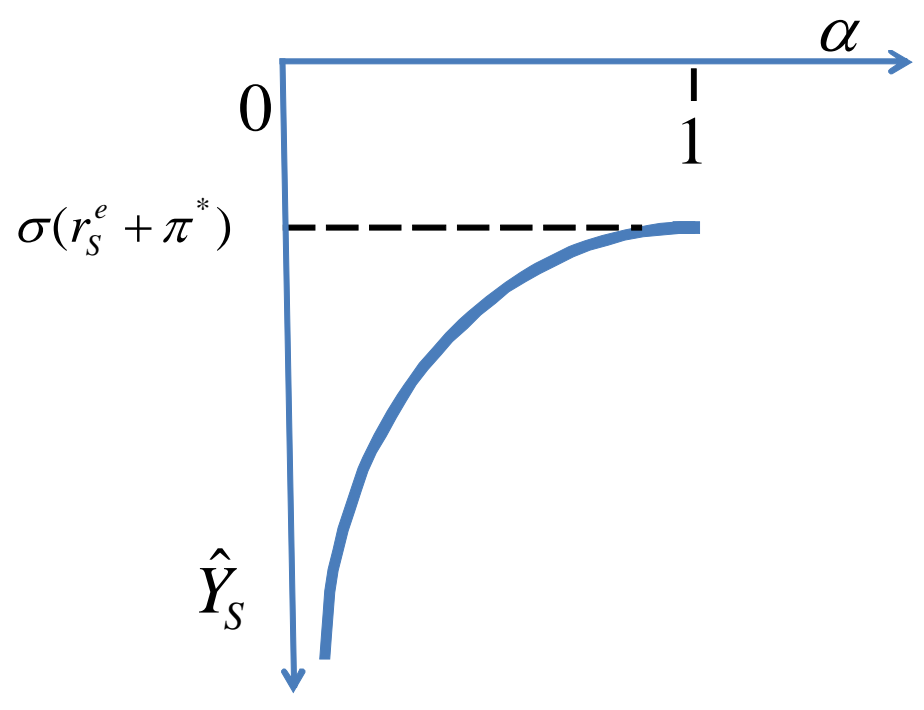

Figure 10: Output in the short run collapses as $\alpha \rightarrow 0$.

The last proposition is illustrated in Figure 10. We see that as the probability $\alpha$ converges to zero, the model explodes. Meanwhile as this probability approaches 1 , output converges to $\sigma\left(r_{S}^{e}+\phi_{\pi}^{-1} \pi_{L}^{*}+\right.$ $\left.\left(1-\phi_{\pi}^{-1}\right) \pi_{M}^{*}\right)$. This proposition above will have important implications for the inflation output trade-off, as we now will see.

\section{The inflation output trade-off revisited}

We now come to the heart of the paper. Let us start with the definition:

Definition 5 The static trade-off between inflation and output is defined in the short, medium and long run as $\frac{d \hat{Y}_{t}}{d \pi_{t}}$ where $t=S, M, L$ and expectations may have adjusted or not. The intertemporal trade-off between expected future inflation and output is defined as $\frac{d \hat{Y}_{S}}{d E_{S} \pi_{M}}, \frac{d \hat{Y}_{S}}{d E_{S} \pi_{L}}$ in the short term and $\frac{d \hat{Y}_{M}^{i}}{d E_{M} \pi_{L}}$ $\left(i=\right.$ high,low) in the medium term, where expected inflation increases due to variations in $\pi_{M}^{*}$ or $\pi_{L}^{*}$ under rational expectations.

The popular discussion of the inflation output trade-off focuses on the trade-off between current inflation and current output. We call this the static trade-off between inflation and output. As we saw already in Section 2 the static trade-off between inflation and output is $\kappa^{-1}$ in the short-run for given inflation expectations. This statistic has the interpretation of telling us how much output is gained in percentage terms for each percentage point increase in inflation. Once inflation expectations have fully adjusted, as is the case in the medium and long-run, in perfect foresight, then there is no static trade-off in the model 
considered thus far.

There is another well defined trade-off between output and inflation in the model which becomes very important once the nominal interest rate hits zero. This is the trade-off between expected inflation in the future and output today. We call this the intertemporal trade-off between inflation and output. In our simple example, this trade-off is given by $\frac{d \hat{Y}_{S}}{d E_{S} \pi_{M}}$ and $\frac{d \hat{Y}_{S}}{d E_{S} \pi_{L}}$ in short run and by $\frac{d \hat{Y}_{M}^{i}}{d E_{M} \pi_{L}}$ in the medium run, where $i=h i g h$, low indexes the state of the world in the medium run depending on whether the shock has turned back to steady state ("high") or not ("low").

Let us first observe that in the absence of the zero bound, the intertemporal trade-off between inflation and output is not of principal importance, at least not in the medium run. To see why, observe first that under our policy rule (16), the central bank will fully offset $r_{t}^{e}$ in the absence of the zero bound. Hence to the extent $r_{t}^{e}$ is the only source of uncertainty, policy can achieve $\hat{Y}_{M}=\hat{Y}_{L}=0$ in the medium and long run for any value of the inflation targets $\pi_{M}^{*}$ and $\pi_{L}^{*}$. Recall that in the medium run we have assumed that inflation expectations have adjusted to policy. In the short-run, however, we take expectations $E_{0} \pi_{S}=0$ as given. This implies some intertemporal trade-off in the short-run as summarized below.

Proposition 6 Suppose A2, $r_{S}^{e}>-\pi_{M}^{*}, \bar{r}>-\pi_{L}^{*}, \phi_{\pi}>1$ and that the nominal interest rate is always positive in the long run. Then the zero bound is not binding, and the intertemporal trade-off between inflation and output in the medium run is $\frac{d \hat{Y}_{M}^{i}}{d E_{M} \pi_{L}}=0$ and it is given by $\frac{d \hat{Y}_{S}}{d E_{S} \pi_{M}}=\frac{\sigma}{1+\kappa \sigma \phi_{\pi}}$ in the short run.

Proof. See Appendix.

To obtain some further intuition for this result consider first the medium run. Note that the shock to the natural rate of interest is small enough so that the zero bound is not binding. This implies that the shock $r_{M}^{e}$ is fully offset, so it makes no difference if it is in the "high" or "low" state - the equilibrium output and inflation is the same. It follows, given our policy rule, that

$$
E_{S} \pi_{M}=\pi_{M}=\phi_{\pi}^{-1} \pi_{L}^{*}+\left(1-\phi_{\pi}^{-1}\right) \pi_{M}^{*}
$$

and $\hat{Y}_{M}=0$, while $i_{M}^{j}=r_{M}^{e}(j)+\pi_{M}^{*}+\phi_{\pi}\left(\pi_{M}-\pi_{M}^{*}\right), j=h i g h$, low. This shows that increasing the long run inflation target has no effect on the medium-run output; it only increases the nominal interest rate in the medium run. This is the first part of the proposition which says there is no intertemporal trade-off in the medium run.

Consider now the effect of increasing the medium or long-term inflation target in the short run - this is the second part of the proposition - while taking the initial expectations as given, i.e. $E_{0} \pi_{S}=0$. The solution then satisfies the two equations

$$
\begin{aligned}
& \hat{Y}_{S}=-\sigma i_{S}+\sigma E_{S} \pi_{M}+\sigma r_{S}^{e}=-\sigma \phi_{\pi} \pi_{S}+\sigma \pi_{M}+\sigma\left(\phi_{\pi}-1\right) \pi_{S}^{*} \\
& \pi_{S}=\kappa \hat{Y}_{S} .
\end{aligned}
$$

The short-run equilibrium determination is plotted up in Figure 11. We see that in the first panel that any increase in medium-term inflation expectations — or in the long-term inflation target, which feeds directly into the medium term via (27) - results in a shift out in demand and thus output. Note that the shift out in demand occurs because an increase in inflation expectations reduces the real interest rate which stimulates spending. This increase is offset to some extent via the increase in the nominal interest rate and thus the increase in demand is less than the increase in inflation expectations depending on the 

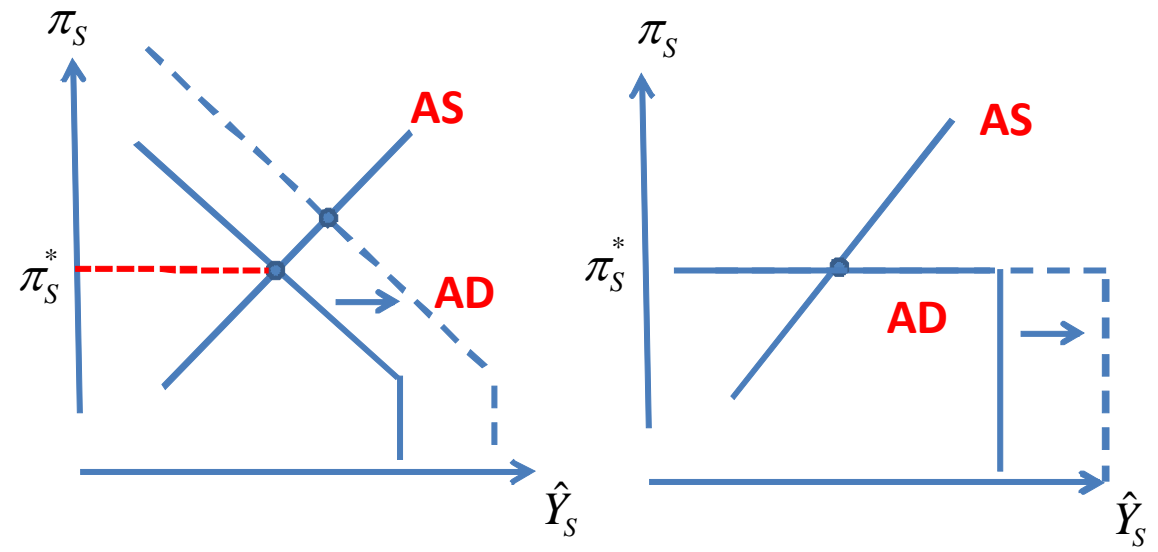

Figure 11: There is no intertemporal tradeoff between expected inflation and output if the central bank is responsive enough in the short run at positive interest rate.

value of $\phi_{\pi}$ which measures the sensitivity of the nominal interest rate to inflation. To make this clear, the second panel shows the case when the central bank strictly targets inflation $\pi_{S}^{*}$ which corresponds to the case $\phi_{\pi} \rightarrow \infty$. In this case we see that the increase in demand due to the increase in medium term inflation expectations is fully offset by the response of the central bank via the nominal interest rate, so that inflation expectations don't change and hence the intertemporal trade-off disappears, i.e., $\lim _{\phi_{\pi} \rightarrow \infty} \frac{\sigma}{1+\sigma \phi_{\pi} \kappa}=0$. To summarize, we see that while in the medium run there is no intertemporal trade-off between inflation and output, such trade-off does exist in the short-run but its size depends entirely on how much short-run inflation the central bank is willing to tolerate. If it targets a particular inflation rate in the short-run then the intertemporal trade-off also disappears.

We now consider the intertemporal trade-off when the zero bound is binding. In that case, not only is the intertemporal trade-off positive in the medium run. In the short-run, instead of possibly disappearing under some parameter configurations, it may become arbitrarily large. A key result of the paper is summarized next.

Proposition 7 Suppose A2, $r_{S}^{e}<-\pi_{M}^{*}, \bar{r}>-\pi_{L}^{*}, \phi_{\pi}>1$ and that the nominal interest rate is always positive in the long run. Then the zero bound is binding and the intertemporal trade-off between medium term output and long term inflation $\frac{d \hat{Y}_{M}^{i}}{d E_{M} \pi_{L}}$, conditional on the zero bound being binding is $\sigma$ ( $\left.i=l o w\right)$, while the intertemporal trade-off between short term output and medium term inflation $\frac{d \hat{Y}_{S}}{d E_{S} \pi_{M}}$ is $\sigma$, and between short-run output and long-run inflation, $\frac{d \hat{Y}_{S}}{d E_{S} \pi_{L}}$, is $\sigma \frac{(1-\alpha) \kappa \sigma \phi_{\pi}+1}{\alpha \phi_{\pi}}$.

Proof. See Appendix. 
In sharp contrast to our previous proposition, we now see that long-term inflation increases mediumterm output conditional on the zero bound binding. This is because with the nominal interest rate pinned at zero in the medium term, output is demand determined and given by

$$
\hat{Y}_{M}^{\text {low }}=\sigma E_{M} \pi_{L}+\sigma r_{S}^{e} .
$$

This makes clear that the intertemporal trade-off in the medium run is given by $\sigma$. Meanwhile, the amount of deflation in the "low" state is given by

$$
\pi_{M}^{l o w}=\frac{\kappa}{\alpha} \hat{Y}_{M}^{l o w}+\pi_{M}^{h i g h}
$$

where we used the fact that $E_{S} \pi_{M}=\alpha \pi_{M}^{\text {high }}+(1-\alpha) \pi_{M}^{\text {low }}$.

Let us now move to the short-run determination of output. It is given by

$$
\hat{Y}_{S}=\sigma E_{S} \pi_{M}+\sigma r_{S}^{e},
$$

and inflation is determined by $\pi_{S}=\kappa \hat{Y}_{S}$. From (29) we see immediately that the intertemporal tradeoff between $\hat{Y}_{S}$ and $E_{S} \pi_{M}$ is again $\sigma$. What is perhaps even more interesting is the trade-off between short term output and long term inflation, i.e., $E_{S} \pi_{L}$. As the proposition shows this trade-off is given by

$\sigma \frac{(1-\alpha) \kappa \sigma \phi_{\pi}+1}{\alpha \phi_{\pi}}>0$ so that higher long-term inflation expectations tend to stimulate short-term output, in the face of the zero lower bound. Note that long-run inflation expectations can be completely controlled by the long-run inflation target, i.e. $E_{S} \pi_{L}=\pi_{L}^{*}$. In the limit, as the probability of exiting the zero bound in the medium term diminishes, i.e., as $\alpha \rightarrow 0$, the trade-off becomes arbitrarily large. This is the opposite of the contractionary spiral we just saw in the last section; it works here in a virtuous direction. The reason is that as $\alpha$ approaches 0 , then a greater deal of deflation is needed in the medium run by equation (28) in order to accommodate a given output gap $\hat{Y}_{M}^{\text {low }}$ (given by $\hat{Y}_{M}^{\text {low }}=\sigma r_{S}^{e}+\sigma E_{M} \pi_{L}$ ). The amount of deflation needed is greater the smaller is $\alpha$, as we saw in Figure 8, which led to a collapse in the short-run. But this also implies that the benefit of increasing long run inflation (and thus $\hat{Y}_{M}^{\text {low }}$ ) is greater the lower is $\alpha$ as shown in Figure 12.

It is worth noting that one does not require inflation to increase permanently to stimulate short-term output, even though we label "long-run inflation" the inflation rate obtained once the shock has subsided. Instead, what is important, is that inflation is increased in the period immediately after the shock has subsided; this is in fact how long-run is defined in our model.

\section{Non-existence of the equilibrium in a non-linear version of the model}

While our analysis has been conducted in the context of a linearized model, it is important to realize that our results are not merely a feature of the linear approximation. In fact, many of the results discussed above carry through in the non-linear model described in Section 3.1. To show this, we briefly mention a few results, leaving the proofs in the Appendix.

The following proposition, which relates to Benhabib, Schmitt-Grohé and Uribe (2001), establishes the existence of multiple long-run steady states. 


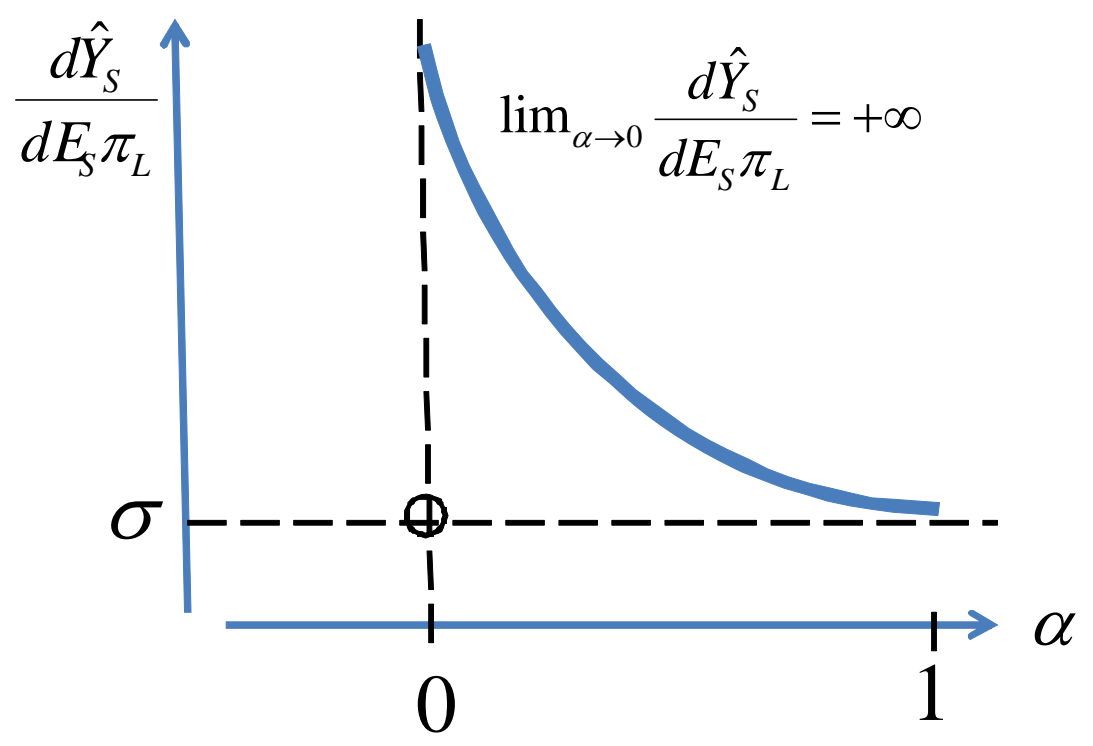

Figure 12: The intertemporal tradeoff between output and expected long term inflation goes to infinity as $\alpha \rightarrow 0$.

Proposition 8 Consider the model given by (6)-(14) for all $t \geq t_{0}$. Suppose that monetary policy is given by an interest-rate reaction function

$$
i_{t}=\phi\left(\Pi_{t}, \Pi_{t}^{*}, \xi_{t}\right)
$$

where $\Pi_{t} \equiv P_{t} / P_{t-1}$ denotes inflation, $\Pi_{t}^{*}$ is the inflation target, and that the policy rule $\phi()$ is non-negative for all values of its arguments, is increasing in its first argument, and that $\partial \phi\left(\Pi_{t}^{*}, \Pi_{t}^{*}, \xi_{t}\right) / \partial \Pi_{t}>1$, so that the "Taylor principle" applies around the inflation target. Suppose furthermore that agents know at some date $t_{1} \geq t_{0}$, that the inflation target $\Pi_{t}^{*}$ will remain constant at $\Pi_{t}^{*}=\Pi^{*}>\beta$ for all $t \geq t_{1}$. Then the model (6)-(14), (30) admits at least two possible steady states characterized by constant values $\bar{Y}_{L}, \bar{C}_{L}, \bar{l}_{L}, \bar{l}_{L}, \bar{\Pi}_{L}, \bar{W}_{L}$ as well as paths for the price levels $\bar{p}_{t}(1), \bar{p}_{t}(2), \bar{P}_{t}$ in all periods $t \geq t_{1}$.

(i) In the first (regular) steady state, the nominal interest rate $\bar{\imath}_{L}$ reaches a positive value $\bar{\imath}_{L}=\phi\left(\Pi_{L}^{*}, \Pi_{L}^{*}, \bar{\xi}_{L}\right)=$ $\Pi_{L}^{*} \beta^{-1}-1>0$, inflation is equal to the target rate $\bar{\Pi}_{L}=\Pi_{L}^{*}$, the price indices $\bar{p}_{t}(1), \bar{p}_{t}(2), \bar{P}_{t}$ grow at rate $\Pi_{L}^{*}$, and $\bar{Y}_{L}=\bar{l}_{L}=\bar{C}_{L}$ and $\bar{W}_{L}$ are given by

$$
\frac{v_{l}\left(\bar{Y}_{L}\right)}{u_{c}\left(\bar{Y}_{L}\right)}=\frac{\theta-1}{\theta}=\bar{W}_{L}
$$

(ii) In the second (Friedman) steady state, the nominal interest rate is at the zero lower bound, $\bar{\imath}_{L}=0$, the steady-state value $\bar{\Pi}_{L}$ shows perpetual deflation at the rate of time preference, $\bar{\Pi}_{L}=\beta$, the price indices (in log) fall at rate $\bar{\Pi}_{L}$, and $\bar{Y}_{L}=\bar{l}_{L}=\bar{C}_{L}$ and $\bar{W}_{L}$ are again given by (31).

Proof. See Appendix.

As stated, at least two long-run steady states are possible in this model: one with constant inflation at the central bank's target level and a positive nominal interest rate, and one with zero nominal interest rate (as in the Friedman rule) and perpetual deflation. Our earlier analysis took the first steady state as 
given. Importantly, however, the lack of existence of a medium run equilibrium does not depend on such an assumption. In fact, as established in the next proposition, if the preference shock takes a low enough value in the medium run, then no medium run equilibrium exists in the non-linear version of the model, under perfect foresight. We modify slightly Assumption A1 as follows:

A1' Consider the three periods $t=S, M, L$. In period $t=S$ there is an unexpected shock $\xi_{S}<\beta \bar{\Pi}_{L}^{-1} \bar{\xi}_{L}$, where $\bar{\xi}_{L}$ is the steady-state value of the preference shock and $\bar{\Pi}_{L}$ is the steady state inflation rate. In period $t=M$ the shock is still $\xi_{M}=\xi_{S}$. In periods $t \geq L$, the shock is back at its steady state $\bar{\xi}_{L}$. While the shock is unexpected in period $t=S$, there is perfect foresight between $S, M$, and $L$. Periods $t>L$ are identical to $L$ : there are no shocks and agents have perfect foresight.

Proposition 9 Under Assumption A1', for any given long-run equilibrium $\bar{Y}_{L}, \bar{C}_{L}, \bar{l}_{L}, \bar{\imath}_{L}, \bar{\Pi}_{L}, \bar{W}_{L}$ satisfying (6)-(30), there exists no medium-term equilibrium for small enough value of the exogenous disturbance $\xi_{M}$, i.e., if

$$
\xi_{M}<\beta \bar{\Pi}_{L}^{-1} \bar{\xi}_{L}
$$

Proof. See Appendix.

The condition (32) causing the absence of any medium-term equilibrium is analogous to the requirement that the natural rate of interest be low enough for the nominal rate to be constrained by the zero lower bound, as seen in our analysis above. It requires $\xi_{M}$ to be low enough, so that the representative consumer finds it preferable to consume less in the present (i.e., in the medium term) than in the future (i.e., the long run). Equivalently, the household prefers to save in the medium term. Doing so, reduces aggregate demand in the medium run, leading firms to lower their prices. This lowers inflation and increases the real interest rate. That increase in the medium-term real rate discourages households further from consuming, thereby leading to a collapse of the economy.

Note that if we are in the regular steady state with $\bar{\Pi}_{L}=\Pi_{L}^{*}$, (and $\beta \bar{\Pi}_{L}^{*-1}<1$ ) then the higher the long-run inflation target $\Pi_{L}^{*}$, the easier it is for the medium-term equilibrium to exist. Indeed, the higher the long-run inflation target, the more difficult it is for the condition (32) to be satisfied, as $\xi_{M}$ needs to fall possibly much below $\bar{\xi}_{L}$ for the medium run equilibrium to cease to exist. Instead, if we are in the "Friedman" steady state, where $\bar{\Pi}_{L}=\beta$, then the medium-run equilibrium ceases to exist and the economy collapses as soon as $\xi_{M}$ falls below the long-run value of $\bar{\xi}_{L}$, regardless of the inflation target.

\section{Inflation output trade-off in the New Keynesian model}

So far we have only studied the inflation output trade-off in the model with New Classical Phillips curve. Part of the motivation for this, was that the consensus about the absence of a long-run inflation output trade-off built from the stagflation experience was largely formed around this specification. More recently, an alternative, the so-called New Keynesian Phillips curve has become more popular, both in quantitative monetary models and in discussions of monetary policy (see, e.g., Woodford (2003), Christiano et al. (2005), Smets and Wouters (2007)). Such a relationship can again be obtained from a log-linear approximation to an optimal price-setting condition in the model described in Section 3.1, but with a different assumption about price setting, e.g., when prices are staggered as in the Calvo model, so that each firm gets to reset 
it price with a fixed probability, regardless of its previous history. The resulting Phillips curve can be expressed as

$$
\pi_{t}=\kappa \hat{Y}_{t}+\beta E_{t} \pi_{t+1}
$$

where the relevant expectations are now the expectations at date $t$ of inflation at date $t+1$.

Within that framework, we confirm the two basic insights shown in the context of the previous model. First that inflation and output may contract dramatically if the nominal interest is constrained by the zero lower bound for a long time, as emphasized by Eggertsson and Woodford (2003). Second, that at zero interest rate, even if the static trade-off between inflation and output is small, once expectations adjust, the intertemporal trade-off can be very large or even explosive.

We start by characterizing the long, medium, and short-term equilibrium in the New Keynesian model. Regarding the long-run equilibrium, we note from (33) that in the long run

$$
\hat{Y}_{L}=\frac{1-\beta}{\kappa} \pi_{L}
$$

so that an increase in long-run inflation is associated with a (bit) of an increase in long-run output. This effect is smaller the closer $\beta$ is to 1 . As in the model considered before, assuming that the long-run nominal interest rate is positive, it follows from the output Euler equation (15) and the policy rule (16) that long-run inflation is equal to the long-run target, $\pi_{L}=\pi_{L}^{*}$, and $i_{L}=r_{L}^{e}+\pi_{L}^{*}$. (The proof of this result is analogous to the proof of Proposition 1.)

Having characterized the long run, we can proceed with the medium-term equilibrium:

Proposition 10 Suppose A2, that $r_{S}^{e}<-\pi_{M}^{*}, \bar{r}>-\pi_{L}^{*}, \phi_{\pi}>1$ and that the nominal interest rate is always positive in the long run $t \geq L$. Then for any $\alpha \in[0,1]$ there exists a unique bounded solution given by

$$
\begin{aligned}
\hat{Y}_{M}^{\text {high }} & =\frac{\frac{1-\beta}{\kappa}-\sigma\left(\beta \phi_{\pi}-1\right)}{\kappa \sigma \phi_{\pi}+1} \pi_{L}^{*}+\frac{\sigma\left(\phi_{\pi}-1\right)}{\kappa \sigma \phi_{\pi}+1} \pi_{M}^{*} \\
\pi_{M}^{\text {high }} & =\frac{1+\kappa \sigma}{\kappa \sigma \phi_{\pi}+1} \pi_{L}^{*}+\frac{\kappa \sigma\left(\phi_{\pi}-1\right)}{\kappa \sigma \phi_{\pi}+1} \pi_{M}^{*} \\
\hat{Y}_{M}^{\text {low }} & =\left(\frac{1-\beta}{\kappa}+\sigma\right) \pi_{L}^{*}+\sigma r_{S}^{e} \\
\pi_{M}^{\text {low }} & =(1+\kappa \sigma) \pi_{L}^{*}+\kappa \sigma r_{S}^{e} .
\end{aligned}
$$

Proof. See Appendix.

Interestingly in this case, and in contrast to the New Classical Phillips curve, the medium-term equilibrium does not depend on the specific value of $\alpha$, the probability of exiting the lower bound in the medium term. This is because the Phillips curve now takes the form

$$
\pi_{M}^{j}=\kappa \hat{Y}_{M}^{j}+\beta \pi_{L}^{*}, \quad j=\text { low, high }
$$

in the medium term, where inflation expectations are pinned down by the long-run inflation target, rather than being affected by $\alpha$. So the medium-term equilibrium exists in this case even if $\alpha=0$ in this model, that is, if the natural rate of interest remains in the low state in the medium run, $r_{M}^{e}=r_{S}^{e}$. The lower the natural rate $r_{S}^{e}$, the lower equilibrium output and inflation in the medium run. The effect is however, not 
as dramatic as in the previous model, since inflation is largely anchored by future inflation expectations, which are here assumed unaffected.

In the short run, however, the probability $\alpha$ does play a role in the equilibrium. ${ }^{13}$ But even if $\alpha=0$, there is a well defined equilibrium given by

$$
\begin{aligned}
& \hat{Y}_{S}=\kappa^{-1}\left((1+\kappa \sigma)^{2}-\beta\right) \pi_{L}^{*}+\sigma(\kappa \sigma+2) r_{S}^{e} \\
& \pi_{S}=\left((1+\kappa \sigma)^{2}+\beta \kappa \sigma\right) \pi_{L}^{*}+\kappa \sigma(\beta+\kappa \sigma+2) r_{S}^{e} .
\end{aligned}
$$

These expressions make clear that a negative value of $r_{S}^{e}$ has even stronger adverse effects on short-run output and inflation than is the case in the medium run. Moreover the adverse effects of a negative $r_{S}^{e}$ on inflation and output in the short term are larger the more flexible prices, i.e., the larger $\kappa$.

Extending the model by adding more periods reveals that the short-term impact on inflation and output grows exponentially with the duration of the binding zero-lower-bound. This is related to the finding by Carlstrom, Fuerst and Paustian (2012) that an interest rate peg has a larger impact on inflation the long the duration of the peg, in a similar model. We thus analyze the behavior of the simple New Keynesian model with discrete periods $t=0,1, \ldots$, and establish the following result.

Proposition 11 Suppose that the shocks are such that $r_{t}^{e}=\underline{r}<\bar{r}$, for all $t=0,1, \ldots, T$, that $r_{t}^{e}=\bar{r}$, for all $t>T$, and that the inflation target is constant $\pi_{t}^{*}=\bar{\pi}^{*}$ for all $t>T$. We assume $\bar{r}>-\bar{\pi}^{*}, \phi_{\pi}>1$. Moreover, we assume that $\underline{r}$ is sufficiently low for the lower bound to bind, that is $\underline{r}+\bar{\pi}^{*}<0$, so that $i_{t}=0$ for all $t=0,1, \ldots T$, and that the nominal interest rate is positive in all periods $t>T$. Then the model (15)-(16) and (33) implies a unique bounded equilibrium given by

$$
\begin{aligned}
& \pi_{t}=\left\{\begin{array}{lr}
-\underline{r}+c_{1}\left(\beta e_{1}\right)^{T-t}+c_{2}\left(\beta e_{2}\right)^{T-t} & \text { for } t=0,1, \ldots T \\
\bar{\pi}^{*} & \text { for } t>T
\end{array}\right. \\
& \hat{Y}_{t}=\left\{\begin{array}{lc}
c_{0}+\frac{\sigma c_{1}}{\beta e_{1}-1}\left(\beta e_{1}\right)^{T-t}+\frac{\sigma c_{2}}{\beta e_{2}-1}\left(\beta e_{2}\right)^{T-t} & \text { for } t=0,1, \ldots T \\
\frac{1-\beta}{\kappa} \bar{\pi}^{*} & \text { for } t>T
\end{array}\right.
\end{aligned}
$$

where $c_{0}, c_{1}, c_{2}, e_{1}, e_{2}$ are constants satisfying $c_{2}<0$ and $0<e_{1}<1<\beta^{-1}<e_{2}$. In this equilibrium, output and inflation initially drop and then revert to the long-run steady state after date $T$. The magnitude of the initial drop in inflation and output grows exponentially with $T$, the duration of the natural rate of interest in its low state $\underline{r}$.

Proof. See Appendix.

While the equilibrium literally continues to exist in the simple New Keynesian model, longer durations of the zero-lower-bound imply ever larger drops in output and inflation, given the dominant terms $c_{2}\left(\beta e_{2}\right)^{T-t}$, so that these drops eventually become unboundedly large as $T-t$ becomes very large. Hence, the model imposes a limit on the duration of the recession to prevent it from exploding. Again, our key result from

${ }^{13}$ The equilibrium conditions in the short run can be obtained by combining

$$
\begin{aligned}
& \pi_{S}=\kappa \hat{Y}_{S}+\beta E_{S} \pi_{M} \\
& \hat{Y}_{S}=\alpha \hat{Y}_{M}^{h i g h}+(1-\alpha) \hat{Y}_{M}^{\text {low }}+\sigma \alpha \pi_{M}^{\text {high }}+\sigma(1-\alpha) \pi_{M}^{\text {low }}+\sigma r_{S}^{e}
\end{aligned}
$$

with the solutions for the medium-term equilibrium listed in Proposition 10. Here we note that $i_{S}=0$, and $E_{S-1} \pi_{S}=0$, since we assume a zero inflation target in period $S$. 
before applies: increasing the inflation target temporarily mitigates the adverse impact of the shock. We now consider the intertemporal trade-off under the assumptions of Proposition 11. The main result is summarized as follows.

Proposition 12 Under the assumptions of Proposition 11, the intertemporal trade-off between output in periods $t=0,1, \ldots T$ and long term inflation is given by

$$
\frac{d \hat{Y}_{t}}{d \bar{\pi}^{*}}=\frac{d c_{0}}{d \bar{\pi}^{*}}+\frac{\sigma}{\beta e_{1}-1} \frac{d c_{1}}{d \bar{\pi}^{*}}\left(\beta e_{1}\right)^{T-t}+\frac{\sigma}{\beta e_{2}-1} \frac{d c_{2}}{d \bar{\pi}^{*}}\left(\beta e_{2}\right)^{T-t}
$$

which is positive for large enough $T$, since $\frac{\sigma}{\beta e_{2}-1}>0, \frac{d c_{2}}{d \bar{\pi}^{*}}>0$ and $\left(\beta e_{2}\right)^{T-t}>1$ is the dominant term. This trade-off grows exponentially with $T$, the duration of the natural rate of interest in its low state $\underline{r}$.

Proof. The result is obtained by differentiating expression in Proposition 11, noting that $e_{1}$ and $e_{2}$ do not depend on $\bar{\pi}^{*}$.

The proposition states that in the New Keynesian the model, the intertemporal trade-off between inflation and output can be arbitrarily large, if the natural rate of interest remains in its low state for a long time.

\section{Conclusion}

We have emphasized the positive trade-off between inflation and output in both a standard New Classical model and a New Keynesian model. In particular, the notion that monetary policy is neutral, once inflation is fully anticipated, is inconsistent with the demand side of standard models of monetary policy if the short-term interest rate is constrained by the zero lower bound. When the shocks are such that the nominal interest rate is constrained by the zero bound, anticipated inflation is far from neutral, with output increasing if inflation is anticipated to rise. Moreover, contrary to the common belief that inflation is less effective at stimulating output when inflation becomes more and more anticipated, the benefits of higher anticipated inflation can be extremely large at the zero bound.

The results presented here suggest that it may be beneficial to let the near-term inflation target move over time in a way that depends on the state of the economy. In particular, our analysis reveals that output and inflation can be stabilized more effectively by raising targeted inflation in the medium run, i.e., in a relatively short period after the shock which brought the economy to the zero bound has subsided. However, as in conventional analyses, once the economy has exited from the zero lower bound, there is no meaningful gain from raising the long-run inflation target.

\section{References}

[1] Barro, R. J., and D. B. Gordon (1983), "A Positive Theory of Monetary Policy in a Natural Rate Model," Journal of Political Economy 91(4), August: 589-610.

[2] Bhattarai, S., Eggertsson G., and R. Schoenle (2012), "Is Increased Price Flexibility Stabilizing? Redux," Federal Reserve Bank of New York Staff Reports 540, January 2012. 
[3] Benhabib, J., S. Schmitt-Grohé, and M. Uribe (2001), "The Perils of Taylor Rules," Journal of Economic Theory 96, 40-69.

[4] Carlstrom, C. T., T. S. Fuerst, and M. Paustian (2012), "How Inflationary Is an Extended Period of Low Interest Rates?" Federal Reserve Bank of Cleveland, working paper no. 12-04.

[5] Christiano, L. , M. Eichenbaum, and C. Evans (2005), "Nominal Rigidities and the Dynamic Effect of a Shock to Monetary Policy," Journal of Political Economy, 113(1): 1-45.

[6] Christiano, L., M. Eichenbaum, and S. Rebelo (2011), "When Is the Government Spending Multiplier Large?," Journal of Political Economy, 119(1): 78-121.

[7] Cochrane, J., (2012), "Woodford and Jackson Hole," at http://johnhcochrane.blogspot.com/2012/09/ woodford-at-jackson-hole.html.

[8] Eggertsson, G. B., and M. Woodford (2003), "The Zero Bound on Interest Rates and Optimal Monetary Policy," Brookings Papers on Economic Activity, 34(2003-1): 139-235.

[9] Eggertsson, G. B. (2012), "Was the New Deal Contractionary?" American Economic Review, 102(1).

[10] Eichengreen, B. and J. Sachs (1985), "Exchange Rates and Economic Recovery in the 1930s," The Journal of Economic History, 45(4): 925-946.

[11] Giannoni, M. P. and M. Woodford (2005), "Optimal Inflation Targeting Rules," in Ben Bernanke and Michael Woodford (eds.), The Inflation-Targeting Debate, Chicago: University of Chicago Press, pp. 93-162.

[12] Goodfriend, M., and R.G. King (2005), "The Incredible Volcker Disinflation," Journal of Monetary Economics, Vol. 52(5): 981-1015.

[13] Krugman, P. (1998), "It's Baaack! Japan's Slump and the return of the Liquidity Trap," Brookings Papers on Economic Activity, 2(1998).

[14] Kydland, F. E., and E. C. Prescott (1977). "Rules Rather than Discretion: The Inconsistency of Optimal Plans." Journal of Political Economy 85: 473-491.

[15] Lucas, R.E. (1972), "Expectations and the Neutrality of Money," Journal of Economic Theory 4, 103-124.

[16] Phelps, E. S. (1967), "Phillips Curves, Expectations of Inflation and Optimal Unemployment over Time," Economica, Vol. 34, 254-281.

[17] Sargent, T. and N. Wallace (1975), "Rational Expectations and the Theory of Economic Policy," Journal of Monetary Economics 2: 169-83.

[18] Sargent, T. J. (1999), The Conquest of American Inflation. Princeton, NJ: Princeton University Press.

[19] Smets, F., and R. Wouters (2007), "Shocks and Frictions in US Business Cycles: A Bayesian DSGE Approach," American Economic Review 97(3): 586-606. 
[20] Werning, I. (2012), "Managing a Liquidity Trap: Monetary and Fiscal Policy," Working Paper, MIT.

[21] Woodford, M. (2003), Interest and Prices: Foundations of a Theory of Monetary Policy. Princeton University Press. 


\section{A Appendix: Proof of Propositions for the New Classical Model}

\section{A.1 Proof of Proposition 2}

Proof. Under the assumptions $\mathrm{A} 1, \bar{r}>-\pi_{L}^{*}, \phi_{\pi}>1$ and that the nominal interest rate is always positive in the long run, it follows from Proposition 1 that a long-run equilibrium is well defined with $\hat{Y}_{L}=0$, and $E_{M} \pi_{L}=\pi_{L}^{*}$. Next, suppose, as a way of contradiction, that a medium run equilibrium $\left\{\pi_{M}, \hat{Y}_{M}, i_{M}\right\}$ satisfying (3) and (15)-(16) exists. Perfect foresight between periods $S$ and $M$ implies $E_{S} \pi_{M}=\pi_{M}$, so that the aggregate supply equation (3) in period $M$ implies $\hat{Y}_{M}=0$. On the demand side, consider two alternative cases. First, if $r_{M}^{e}+\pi_{M}^{*}+\phi_{\pi}\left(\pi_{M}-\pi_{M}^{*}\right) \leq 0$, then $i_{M}=0$ by (16). Since $r_{M}^{e}+\pi_{L}^{*}<0$ by assumption, it follows from (15) that $\hat{Y}_{M}=\sigma\left(\pi_{L}^{*}+r_{M}^{e}\right)<0$. Alternatively, if $r_{M}^{e}+\pi_{M}^{*}+\phi_{\pi}\left(\pi_{M}-\pi_{M}^{*}\right)>0$, then $i_{M}>0$ by (16). In this case, (15) implies

$$
\hat{Y}_{M}=-\sigma\left(i_{M}-\pi_{L}^{*}-r_{M}^{e}\right)=-\sigma i_{M}+\sigma\left(\pi_{L}^{*}+r_{M}^{e}\right)<0 .
$$

So in both cases (15)-(16) imply that $\hat{Y}_{M}<0$. This leads to a contradiction with the implication of (3) according to which $\hat{Y}_{M}=0$. Hence no medium run equilibrium $\left\{\pi_{M}, \hat{Y}_{M}, i_{M}\right\}$ satisfying (3) and (15)-(16) exists.

\section{A.2 Proof of Proposition 3}

Proof. Under the assumptions A2, $\bar{r}>-\pi_{L}^{*}, \phi_{\pi}>1$ and that the nominal interest rate is always positive in the long run, it follows from Proposition 1 that $\hat{Y}_{L}=0$. Using this, equations (19)-(24) can be written in matrix form as

$$
\left[\begin{array}{cccccccc}
\alpha & -\alpha & -\kappa & 0 & 0 & 0 & 0 & 0 \\
-(1-\alpha) & 1-\alpha & 0 & -\kappa & 0 & 0 & 0 & 0 \\
0 & 0 & 1 & 0 & \sigma & 0 & 0 & 0 \\
0 & 0 & 0 & 1 & 0 & \sigma & 0 & 0 \\
0 & 0 & 0 & 0 & 1 & 0 & 0 & 0 \\
0 & -\phi_{\pi} & 0 & 0 & 0 & 1 & 0 & 0 \\
0 & 0 & 0 & 0 & 0 & 0 & 1 & -\kappa \\
-\sigma(1-\alpha) & -\sigma \alpha & -(1-\alpha) & -\alpha & 0 & 0 & 0 & 1
\end{array}\right]\left[\begin{array}{c}
\pi_{M}^{\text {low }} \\
\pi_{M}^{h i g h} \\
\hat{Y}_{M}^{\text {low }} \\
\hat{Y}_{M}^{\text {high }} \\
i_{M}^{\text {low }} \\
i_{M}^{\text {high }} \\
\pi_{S} \\
\hat{Y}_{S}
\end{array}\right]=\left[\begin{array}{c}
0 \\
0 \\
\sigma\left(\pi_{L}^{*}+r_{S}^{e}\right) \\
\sigma\left(\pi_{L}^{*}+r_{L}^{e}\right) \\
0 \\
r_{L}^{e}-\left(\phi_{\pi}-1\right) \pi_{M}^{*} \\
0 \\
\sigma r_{S}^{e}
\end{array}\right]
$$

We note that the determinant of the matrix on the left is $\alpha \kappa \sigma \phi_{\pi}$. So, provided that $\alpha>0$ and that $\kappa \sigma \phi_{\pi}>0$, we can invert the matrix to obtain the solution expressed in the proposition.

\section{A.3 Proof of Proposition 6}

Proof. Under the assumptions A2, $\bar{r}>-\pi_{L}^{*}, \phi_{\pi}>1$ and that the nominal interest rate is always positive in the long run, it follows from Proposition 1 that $\hat{Y}_{L}=0$ and that $\pi_{L}=\pi_{L}^{*}$. The assumption $r_{S}^{e}>-\pi_{M}^{*}$ furthermore implies that the nominal interest rate is not constrained by the zero bound in the short term. Using this, the equations characterizing the short and medium term equilibrium are given by (19)-(21), 
(24) and

$$
\begin{aligned}
i_{M}^{\text {low }} & =r_{S}^{e}+\pi_{M}^{*}+\phi_{\pi}\left(\pi_{M}^{l o w}-\pi_{M}^{*}\right) \\
i_{M}^{\text {high }} & =r_{L}^{e}+\pi_{M}^{*}+\phi_{\pi}\left(\pi_{M}^{\text {high }}-\pi_{M}^{*}\right) \\
i_{S} & =r_{S}^{e}+\pi_{S}^{*}+\phi_{\pi}\left(\pi_{S}-\pi_{S}^{*}\right) \\
\hat{Y}_{S} & =\alpha \hat{Y}_{M}^{\text {high }}+(1-\alpha) \hat{Y}_{M}^{\text {low }}-\sigma i_{S}+\sigma \alpha \pi_{M}^{\text {high }}+\sigma(1-\alpha) \pi_{M}^{l o w}+\sigma r_{S}^{e} .
\end{aligned}
$$

Combining the last two equations yields

$$
\hat{Y}_{S}=\alpha \hat{Y}_{M}^{h i g h}+(1-\alpha) \hat{Y}_{M}^{l o w}-\sigma\left(\pi_{S}^{*}+\phi_{\pi}\left(\pi_{S}-\pi_{S}^{*}\right)\right)+\sigma \alpha \pi_{M}^{\text {high }}+\sigma(1-\alpha) \pi_{M}^{l o w} .
$$

Equations (19)-(21), (24), (34)-(35), (38) can in turn be written in matrix form as

$$
\left[\begin{array}{cccccccc}
\alpha & -\alpha & -\kappa & 0 & 0 & 0 & 0 & 0 \\
-(1-\alpha) & 1-\alpha & 0 & -\kappa & 0 & 0 & 0 & 0 \\
0 & 0 & 1 & 0 & \sigma & 0 & 0 & 0 \\
0 & 0 & 0 & 1 & 0 & \sigma & 0 & 0 \\
-\phi_{\pi} & 0 & 0 & 0 & 1 & 0 & 0 & 0 \\
0 & -\phi_{\pi} & 0 & 0 & 0 & 1 & 0 & 0 \\
0 & 0 & 0 & 0 & 0 & 0 & 1 & -\kappa \\
-\sigma(1-\alpha) & -\sigma \alpha & -(1-\alpha) & -\alpha & 0 & 0 & \sigma \phi_{\pi} & 1
\end{array}\right]\left[\begin{array}{c}
\pi_{M}^{\text {low }} \\
\pi_{M}^{\text {high }} \\
\hat{Y}_{M}^{\text {low }} \\
\hat{Y}_{M}^{\text {high }} \\
i_{M}^{\text {low }} \\
i_{M}^{\text {high }} \\
\pi_{S} \\
\hat{Y}_{S}
\end{array}\right]=\left[\begin{array}{c}
0 \\
0 \\
\sigma\left(\pi_{L}^{*}+r_{S}^{e}\right) \\
\sigma\left(\pi_{L}^{*}+r_{L}^{e}\right) \\
r_{S}^{e}-\left(\phi_{\pi}-1\right) \pi_{M}^{*} \\
r_{L}^{e}-\left(\phi_{\pi}-1\right) \pi_{M}^{*} \\
0 \\
\sigma\left(\phi_{\pi}-1\right) \pi_{S}^{*}
\end{array}\right] .
$$

We note that the determinant of the matrix on the left is $\kappa \sigma \phi_{\pi}\left(\kappa \sigma \phi_{\pi}+1\right)^{2}$. So, provided that $\kappa \sigma \phi_{\pi}>0$, we can invert the matrix to obtain the solution

$$
\begin{aligned}
\pi_{M} & =\pi_{M}^{l o w}=\pi_{M}^{h i g h}=\frac{1}{\phi_{\pi}}\left(\pi_{L}^{*}+\left(\phi_{\pi}-1\right) \pi_{M}^{*}\right) \\
\hat{Y}_{M} & =\hat{Y}_{M}^{\text {low }}=\hat{Y}_{M}^{h i g h}=0 \\
i_{M}^{l o w} & =\pi_{L}^{*}+r_{S}^{e} \\
i_{M}^{h i g h} & =\pi_{L}^{*}+r_{L}^{e} \\
\hat{Y}_{S} & =\frac{\sigma}{\phi_{\pi}\left(1+\kappa \sigma \phi_{\pi}\right)}\left(\pi_{L}^{*}+\left(\phi_{\pi}-1\right) \pi_{M}^{*}+\phi_{\pi}\left(\phi_{\pi}-1\right) \pi_{S}^{*}\right) \\
\pi_{S} & =\kappa \hat{Y}_{S}
\end{aligned}
$$

Given that the expression for $\hat{Y}_{S}$ can be rewritten as $\hat{Y}_{S}=\frac{\sigma}{\left(1+\kappa \sigma \phi_{\pi}\right)}\left(\pi_{M}+\left(\phi_{\pi}-1\right) \pi_{S}^{*}\right)$, and that $\hat{Y}_{M}=0$, the trade-offs are given by $\frac{d \hat{Y}_{S}}{d E_{S} \pi_{M}}=\frac{\sigma}{\left(1+\kappa \sigma \phi_{\pi}\right)}$ and $\frac{d \hat{Y}_{M}^{i}}{d E_{M} \pi_{L}}=0$.

\section{A.4 Proof of Proposition 7}

Proof. Given the assumptions, Proposition 3 applies and determines the short-run and medium-run equilibrium. Differentiating the expressions in Proposition 3 and noting that long-run inflation satisfies 
$\pi_{L}=E_{S} \pi_{L}=E_{M} \pi_{L}=\pi_{L}^{*}$, we obtain the trade-offs:

$$
\begin{aligned}
& \left.\frac{d \hat{Y}_{M}^{\text {low }}}{d\left(E_{S} \pi_{L}\right)}\right|_{\substack{d \pi_{M}^{*}=d \pi_{L}^{*} \\
d r_{S}^{e}=d r_{L}^{*}=0}}=\left.\frac{d \hat{Y}_{M}^{l o w}}{d \pi_{L}^{*}}\right|_{\substack{d \pi_{M}^{*}=d \pi_{L}^{*} \\
d r_{S}^{e}=d r_{L}^{e}=0}}\left(\frac{d E_{S} \pi_{L}}{d \pi_{L}^{*}}\right)^{-1}=\sigma>0 \\
& \left.\frac{d \hat{Y}_{S}}{d\left(E_{S} \pi_{M}\right)}\right|_{\substack{d \pi=d \pi_{M}^{*}=d \pi_{L}^{*} \\
d r_{S}^{e}=d r_{L}^{e}=0}}=\left.\frac{d \hat{Y}_{s}}{d \pi}\left(\frac{d E_{S} \pi_{M}}{d \pi}\right)^{-1}\right|_{\begin{array}{c}
d \pi=d \pi_{M}^{*}=d \pi_{L}^{*} \\
d r_{S}^{e}=d r_{L}^{e}=0
\end{array}}=\left.\frac{d \hat{Y}_{s}}{d \pi}\left(\alpha \frac{d \pi_{M}^{h i g h}}{d \pi}+(1-\alpha) \frac{d \pi_{M}^{l o w}}{d \pi}\right)^{-1}\right|_{\begin{array}{c}
d \pi=d \pi_{M}^{*}=d \pi_{L}^{*} \\
d r_{S}^{e}=d r_{L}^{e}=0
\end{array}} \\
& =\left(\sigma \frac{(1-\alpha) \phi_{\pi} \kappa \sigma+1}{\phi_{\pi} \alpha}+\sigma \frac{\phi_{\pi}-1}{\phi_{\pi}}\right)\left(\alpha\left(\frac{1}{\alpha \phi_{\pi}}+\frac{\phi_{\pi}-1}{\phi_{\pi}}\right)+(1-\alpha)\left(\frac{1+\phi_{\pi} \kappa \sigma}{\phi_{\pi} \alpha}+\frac{\phi_{\pi}-1}{\phi_{\pi}}\right)\right)^{-1} \\
& =\sigma>0 \\
& \left.\frac{d \hat{Y}_{S}}{d E_{S} \pi_{L}}\right|_{\substack{d \pi=d \pi_{M}^{*}=d \pi_{L}^{*} \\
d r_{S}^{e}=d r_{L}^{e}=0}}=\frac{d \hat{Y}_{S}}{d \pi_{L}^{*}}=\sigma \frac{(1-\alpha) \phi_{\pi} \kappa \sigma+1}{\phi_{\pi} \alpha} .
\end{aligned}
$$

\section{B Appendix: Nonlinear Model}

\section{B.1 Model}

The nonlinear model described in the text is repeated here for convenience using (13) to eliminate $C_{t}$ and $l_{t}$. The household's optimal conditions for consumption and leisure are given by

$$
\begin{aligned}
u_{c}\left(Y_{t}\right) \xi_{t} & =\left(1+i_{t}\right) \beta E_{t}\left[u_{c}\left(Y_{t+1}\right) \xi_{t+1} \Pi_{t+1}^{-1}\right] \\
W_{t} & =\frac{v_{l}\left(Y_{t}\right)}{u_{c}\left(Y_{t}\right)}
\end{aligned}
$$

where

$$
\Pi_{t} \equiv \frac{P_{t}}{P_{t-1}}
$$

and the nominal interest rate satisfies the zero lower bound constraint

$$
i_{t} \geq 0
$$

The firms' optimal conditions for pricing are given by

$$
\frac{p_{t}(1)}{P_{t}}=\frac{\theta}{\theta-1} W_{t}
$$

and

$$
E_{t-1}\left[u_{c}\left(Y_{t}\right) Y_{t}\left(\frac{p_{t}(2)}{P_{t}}\right)^{-\theta}\left(\frac{p_{t}(2)}{P_{t}}-\frac{\theta}{\theta-1} W_{t}\right)\right]=0,
$$

and the aggregate price level satisfies

$$
1=\gamma \frac{p_{t}(1)}{P_{t}}+(1-\gamma) \frac{p_{t}(2)}{P_{t}}
$$

Finally government policy is given by an interest rate reaction function

$$
i_{t}=\phi\left(\Pi_{t}, \Pi_{t}^{*}, \xi_{t}\right)
$$


where we assume that the function $\phi()$ is non-negative for all values of its arguments (otherwise policy would not be feasible given the zero bound) and increasing in $\Pi_{t}$. In addition, fiscal policy is assumed to be such that (6) and (7) are satisfied. An equilibrium can now be defined as a set of stochastic processes $\left\{\frac{p_{t}(1)}{P_{t}}, \frac{p_{t}(2)}{P_{t}}, \Pi_{t}, Y_{t}, W_{t}, i_{t}\right\}$ and a fiscal policy that satisfy equations (6)-(7), (39)-(46) in all periods $t \geq t_{0}$, for given $\left\{\xi_{t}, \Pi_{t}^{*}\right\}$.

\section{B.2 Long-run steady state: Proof of Proposition 8}

Proof. Consider the long-run steady states given by constant values $\bar{Y}_{L}, \bar{\imath}_{L}, \bar{\Pi}_{L}, \bar{W}_{L}$, as well as paths for the price levels $\bar{p}_{t}(1), \bar{p}_{t}(2), \bar{P}_{t}$ for given exogenous variables $\bar{\xi}_{L}, \bar{\Pi}_{L}^{*}$ that satisfy the above equations in all periods $t \geq t_{1}$. Optimal pricing conditions for all $t \geq t_{1}$ are given by

$$
\frac{\bar{p}_{t}(1)}{\bar{P}_{t}}=\frac{\theta}{\theta-1} \bar{W}_{L}
$$

and

$$
\frac{\bar{p}_{t}(2)}{\bar{P}_{t}}=\frac{\theta}{\theta-1} \bar{W}_{L}
$$

given that $u_{c}\left(\bar{Y}_{L}\right) \bar{Y}_{L}\left(\bar{p}_{t}(2) / \bar{P}_{t}\right)^{-\theta}>0$ by assumption. The two optimal pricing equations combined with the aggregate price level equation $(45)$

$$
1=\gamma \frac{\bar{p}_{t}(1)}{\bar{P}_{t}}+(1-\gamma) \frac{\bar{p}(2)}{\bar{P}_{t}}
$$

imply

$$
\bar{P}_{t}=\bar{p}_{t}(1)=\bar{p}_{t}(2)
$$

and

$$
\bar{W}_{L}=\frac{\theta-1}{\theta} .
$$

Next, combining this with (40) determines implicitly steady-state output using

$$
\frac{v_{l}\left(\bar{Y}_{L}\right)}{u_{c}\left(\bar{Y}_{L}\right)}=\frac{\theta-1}{\theta} .
$$

To determine steady-state inflation, we use the consumption Euler equation (39) at the steady state

$$
u_{c}\left(\bar{Y}_{L}\right) \bar{\xi}_{L}=\left(1+\bar{\imath}_{t}\right) \beta u_{c}\left(\bar{Y}_{L}\right) \bar{\xi}_{L} \bar{\Pi}_{t+1}^{-1}
$$

for any $t \geq t_{1}$. This simplifies to

$$
\beta^{-1}=\left(1+\bar{\imath}_{t}\right) \bar{\Pi}_{t+1}^{-1} .
$$

Combining this with the government's interest-rate reaction function

$$
\bar{\imath}_{t}=\phi\left(\bar{\Pi}_{t}, \bar{\Pi}_{t}^{*}, \bar{\xi}_{t}\right)
$$

we get

$$
\bar{\Pi}_{t+1}=\beta\left(1+\phi\left(\bar{\Pi}_{t}, \bar{\Pi}_{t}^{*}, \bar{\xi}_{t}\right)\right),
$$

for any $t \geq t_{1}$. As analyzed in Benhabib, Schmitt-Grohé and Uribe (2001), this equation admits at least two constant solutions. One solution $\bar{\Pi}_{t}=\bar{\Pi}_{t}^{*}$, and $\bar{\imath}_{t}=\phi\left(\bar{\Pi}_{t}^{*}, \bar{\Pi}_{t}^{*}, \bar{\xi}_{t}\right)=\beta^{-1} \bar{\Pi}_{t}^{*}-1>0$, for all $t \geq t_{1}$. The other constant solution is $\bar{\Pi}_{t}=\beta<1$ and $\bar{\imath}_{t}=\phi\left(\beta, \bar{\Pi}_{t}^{*}, \bar{\xi}_{t}\right)=0$, for all $t \geq t_{1}$. As shown in Woodford (2003, chap. 2), for any initial inflation $\Pi_{0} \in\left(0, \bar{\Pi}_{L}^{*}\right)$, one of these steady states will eventually be reached. In contrast, for any $\Pi_{0}>\bar{\Pi}_{L}^{*}$, inflation will increase forever and get unboundedly large. Such an equilibrium is not consistent with a constant steady state inflation. 


\section{B.3 Non-existence of medium term equilibrium: Proof of Proposition 9}

Proof. Given Assumption 1', there is perfect foresight between periods $M$ and $L$, so that the optimal pricing conditions (43)-(44) simplify to

$$
\frac{p_{M}(1)}{P_{M}}=\frac{\theta}{\theta-1} W_{M}
$$

and

$$
\frac{p_{M}(2)}{P_{M}}=\frac{\theta}{\theta-1} W_{M},
$$

given that $u_{c}\left(Y_{M}\right) Y_{M}\left(p_{M}(2) / P_{M}\right)^{-\theta}>0$ by assumption. Combining this with (45) yields

$$
\begin{aligned}
P_{M} & =p_{M}(1)=p_{M}(2) \\
W_{M} & =\frac{\theta-1}{\theta} .
\end{aligned}
$$

Using (40), we can determine medium-term output supplied

$$
\frac{v_{l}\left(Y_{M}\right)}{u_{c}\left(Y_{M}\right)}=\frac{\theta-1}{\theta}
$$

so that

$$
Y_{M}=\bar{Y}_{L}
$$

On the demand side, however, equilibrium output must satisfy (39) or

$$
u_{c}\left(Y_{M}\right) \xi_{M}=\left(1+i_{M}\right) \beta u_{c}\left(\bar{Y}_{L}\right) \bar{\xi}_{L} \bar{\Pi}_{L}^{-1} .
$$

Using (50), this simplifies to

$$
\xi_{M}=\left(1+i_{M}\right) \beta \bar{\xi}_{L} \bar{\Pi}_{L}^{-1}
$$

so that $i_{M}<0$, if condition (32) holds. It follows that the zero-lower bound condition (42) is violated and hence that there is no medium-term equilibrium if (32) holds.

\section{Appendix: Proof of Propositions for the New Keynesian Model}

\section{C.1 Proof of Proposition 10}

Proof. Under the assumptions A2, $\bar{r}>-\pi_{L}^{*}, \phi_{\pi}>1$ and that the nominal interest rate is always positive in the long run, the output level in the long run is given by $\hat{Y}_{L}=\frac{1-\beta}{\kappa} \pi_{L}$, and $\pi_{L}=\pi_{L}^{*}, i_{L}=r_{L}^{e}+\pi_{L}^{*}$, as discussed in the text. In addition, we have $E_{t} \hat{Y}_{L}=\frac{1-\beta}{\kappa} \pi_{L}^{*}, E_{t} \pi_{L}=E_{t} \pi_{L+1}=\pi_{L}^{*}$, for any $t=S, M, L$. Moving to the medium run, we have two possible states, i) that the shock reverts back to steady state $r_{M}^{e}=r_{L}^{e}>0$ (which we call "high") and ii) that the shock remains at $r_{M}^{e}=r_{S}^{e}<-\pi^{*}$. The model then solves the following six equations in the medium run

$$
\begin{aligned}
\pi_{M}^{\text {low }} & =\kappa \hat{Y}_{M}^{\text {low }}+\beta \pi_{L}^{*} \\
\pi_{M}^{\text {high }} & =\kappa \hat{Y}_{M}^{\text {high }}+\beta \pi_{L}^{*} \\
Y_{M}^{\text {low }} & =\hat{Y}_{L}-\sigma\left(i_{M}^{\text {low }}-\pi_{L}^{*}-r_{S}^{e}\right) \\
Y_{M}^{\text {high }} & =\hat{Y}_{L}-\sigma\left(i_{M}^{\text {high }}-\pi_{L}^{*}-r_{L}^{e}\right) \\
i_{M}^{\text {low }} & =0 \\
i_{M}^{\text {high }} & =r_{L}^{e}+\pi_{M}^{*}+\phi_{\pi}\left(\pi_{M}^{\text {high }}-\pi_{M}^{*}\right) .
\end{aligned}
$$


These equilibrium conditions can be written in matrix form as

$$
\left[\begin{array}{cccccc}
1 & 0 & -\kappa & 0 & 0 & 0 \\
0 & 1 & 0 & -\kappa & 0 & 0 \\
0 & 0 & 1 & 0 & \sigma & 0 \\
0 & 0 & 0 & 1 & 0 & \sigma \\
0 & 0 & 0 & 0 & 1 & 0 \\
0 & -\phi_{\pi} & 0 & 0 & 0 & 1
\end{array}\right]\left[\begin{array}{c}
\pi_{M}^{\text {low }} \\
\pi_{M}^{\text {high }} \\
\hat{Y}_{M}^{\text {low }} \\
\hat{Y}_{M}^{\text {high }} \\
i_{M}^{\text {low }} \\
i_{M}^{\text {high }}
\end{array}\right]=\left[\begin{array}{c}
\beta \pi_{L}^{*} \\
\beta \pi_{L}^{*} \\
\frac{1-\beta}{\kappa} \pi_{L}^{*}+\sigma \pi_{L}^{*}+\sigma r_{S}^{e} \\
\frac{1-\beta}{\kappa} \pi_{L}^{*}+\sigma \pi_{L}^{*}+\sigma r_{L}^{e} \\
0 \\
r_{L}^{e}-\left(\phi_{\pi}-1\right) \pi_{M}^{*}
\end{array}\right]
$$

We note that the determinant of the matrix on the left hand side is $1+\kappa \sigma \phi_{\pi}>0$. So we can invert that matrix to express the equilibrium in the medium term as

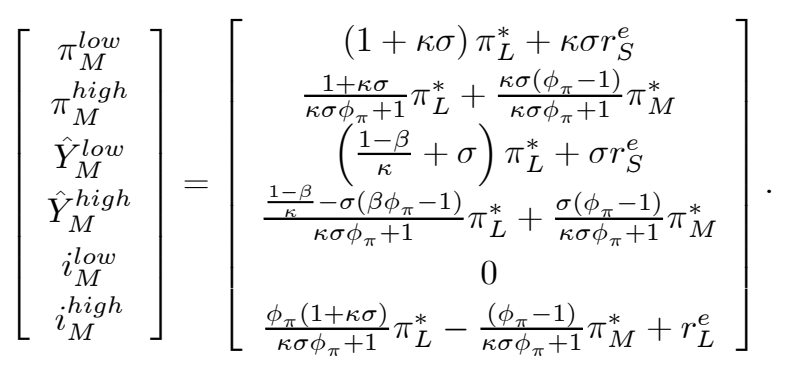

\section{C.2 Proof of Proposition 11}

We start by characterizing the long-run steady state. We next describe the equilibrium after period $T$ and finish with a description of the equilibrium for dates $t=0,1, \ldots T$.

Long-run steady state. The model given by equations (15)-(16) and (33) admits a long-run steady state $\bar{\pi}, \bar{Y}, \bar{\imath}, \bar{r}^{e}$ that satisfies

$$
\begin{aligned}
\bar{\imath} & =\bar{\pi}+\bar{r} . \\
\bar{\imath} & =\max \left\{0, \bar{r}+\bar{\pi}^{*}+\phi_{\pi}\left(\bar{\pi}-\bar{\pi}^{*}\right)\right\} \\
(1-\beta) \bar{\pi} & =\kappa \bar{Y}
\end{aligned}
$$

Combining the first two equations and using the assumption $\bar{\imath}>0$, we obtain

$$
\bar{\pi}+\bar{r}=\bar{r}+\bar{\pi}^{*}+\phi_{\pi}\left(\bar{\pi}-\bar{\pi}^{*}\right)
$$

which simplifies to $\left(\phi_{\pi}-1\right)\left(\bar{\pi}-\bar{\pi}^{*}\right)=0$, and hence to $\bar{\pi}=\bar{\pi}^{*}$, since $\phi_{\pi}>1$. It follows that the steady-state level of output $\left(\hat{Y}_{t}\right)$ is given by $\bar{Y}=\frac{1-\beta}{\kappa} \bar{\pi}^{*}$.

Equilibrium after period $T$. After period $T$, the equations (15)-(16) and (33) reduce to

$$
\begin{aligned}
\pi_{t} & =\kappa \hat{Y}_{t}+\beta E_{t} \pi_{t+1} \\
\hat{Y}_{t} & =E_{t} \hat{Y}_{t+1}-\sigma\left(i_{t}-E_{t} \pi_{t+1}-\bar{r}\right) \\
i_{t} & =\bar{r}+\bar{\pi}^{*}+\phi_{\pi}\left(\pi_{t}-\bar{\pi}^{*}\right),
\end{aligned}
$$


using the assumption $i_{t} \geq 0$ for all $t>T$. Combining the last two equations to eliminate $i_{t}$, we can rewrite the system in matrix form as

$$
\left[\begin{array}{ll}
1 & 0 \\
\sigma & 1
\end{array}\right] E_{t}\left[\begin{array}{c}
\pi_{t+1} \\
\hat{Y}_{t+1}
\end{array}\right]=\left[\begin{array}{cc}
\beta^{-1} & -\beta^{-1} \kappa \\
\sigma \phi_{\pi} & 1
\end{array}\right]\left[\begin{array}{c}
\pi_{t} \\
\hat{Y}_{t}
\end{array}\right]+\left[\begin{array}{c}
0 \\
\sigma\left(1-\phi_{\pi}\right) \bar{\pi}^{*}
\end{array}\right]
$$

or

$$
E_{t} z_{t+1}=A z_{t}+\delta
$$

where

$$
z_{t} \equiv\left[\begin{array}{c}
\pi_{t} \\
\hat{Y}_{t}
\end{array}\right], \quad \delta \equiv\left[\begin{array}{ll}
1 & 0 \\
\sigma & 1
\end{array}\right]^{-1}\left[\begin{array}{c}
0 \\
\sigma\left(1-\phi_{\pi}\right) \pi_{t}^{*}
\end{array}\right]=\left[\begin{array}{c}
0 \\
\sigma\left(1-\phi_{\pi}\right) \bar{\pi}^{*}
\end{array}\right]
$$

and

$$
A=\left[\begin{array}{ll}
1 & 0 \\
\sigma & 1
\end{array}\right]^{-1}\left[\begin{array}{cc}
\beta^{-1} & -\beta^{-1} \kappa \\
\sigma \phi_{\pi} & 1
\end{array}\right]=\left[\begin{array}{cc}
\beta^{-1} & -\beta^{-1} \kappa \\
\sigma\left(\phi_{\pi}-\beta^{-1}\right) & 1+\beta^{-1} \kappa \sigma
\end{array}\right] .
$$

Note that with $\phi_{\pi}>1$, we have $\operatorname{det}(A)=\beta^{-1}\left(\kappa \sigma \phi_{\pi}+1\right)>1$, and $\operatorname{tr}(A)=\beta^{-1}+\beta^{-1} \kappa \sigma+1$. This implies: $\operatorname{det}(A)-\operatorname{tr}(A)=-1+\beta^{-1} \kappa \sigma\left(\phi_{\pi}-1\right)>-1$, and $\operatorname{det}(A)+\operatorname{tr}(A)=\frac{1}{\beta}\left(\beta+\kappa \sigma\left(\phi_{\pi}+1\right)+2\right)>1$. It then follows from Proposition C.1. in Woodford (2003, p. 670) that the matrix $A$ has both eigenvalues outside the unit circle, so that $A^{-1}$ exists and has both eigenvalues inside the unit circle.

Iterating forward equation (51), we have

$$
\begin{aligned}
z_{t} & =-A^{-1} \delta_{t}+E_{t} A^{-1} z_{t+1} \\
& =E_{t}\left(-A^{-1} \delta-A^{-2} \delta \ldots-A^{-n} \delta+A^{-n} z_{t+n}\right) .
\end{aligned}
$$

Since $\lim _{n \rightarrow \infty} E_{t} A^{-n} z_{t+n}=0$ for any bounded process $\left\{z_{t}\right\}$, we obtain the unique bounded solution

$$
z_{t}=-\sum_{T=t}^{\infty} A^{-(T-t+1)} \delta=-A^{-1}\left(I-A^{-1}\right)^{-1} \delta=(I-A)^{-1} \delta=\left[\begin{array}{c}
\bar{\pi}^{*} \\
\frac{1-\beta}{\kappa} \bar{\pi}^{*}
\end{array}\right],
$$

so that inflation and output are at their long-run steady state at all dates $t>T$.

Equilibrium in periods $t=0,1, \ldots, T$. With the zero lower bound assumed binding in periods $t=$ $0,1, \ldots, T$, the model reduces to

$$
\begin{aligned}
& \pi_{t}=\kappa \hat{Y}_{t}+\beta E_{t} \pi_{t+1} \\
& \hat{Y}_{t}=E_{t} \hat{Y}_{t+1}+\sigma\left(E_{t} \pi_{t+1}+\underline{r}\right) .
\end{aligned}
$$

Combining the last two equations yields

$$
E_{t}\left[\pi_{t}-(1+\beta+\kappa \sigma) \pi_{t+1}+\beta \pi_{t+2}\right]=\kappa \sigma \underline{\underline{r}} .
$$

Let $\underline{\pi}$ be the "steady state" value of $\pi_{t}$, in periods $t=0, \ldots T$. It satisfies $\underline{\pi}-(1+\beta+\kappa \sigma) \underline{\pi}+\beta \underline{\pi}=\kappa \sigma \underline{r}$, or $\underline{\pi}=-\underline{r}$. We can then rewrite (53) as the homogenous equation

$$
E_{t}\left[B(L) \tilde{\pi}_{t+2}\right]=0
$$

where $\tilde{\pi}_{t} \equiv \pi_{t}-\underline{\pi}$, the lag polynomial $B(L)$ is given by

$$
\begin{aligned}
B(L) & \equiv L^{2}-(1+\beta+\kappa \sigma) L+\beta \\
& =\beta\left(1-e_{1} L\right)\left(1-e_{2} L\right)
\end{aligned}
$$


and $e_{1}, e_{2}$ are the two roots of the characteristic polynomial $P(x)=\beta x^{2}-(1+\beta+\kappa \sigma) x+1$. Note that $P(x)$ is convex, and $P(0)=1>0, P(1)=-\kappa \sigma<0, P\left(\beta^{-1}\right)=-\kappa \sigma \beta^{-1}<0$, so that $P(x)=0$ admits two real solutions $0<e_{1}<1<\beta^{-1}<e_{2}$. Expanding (55) and comparing it to $B(L)$ reveals that $e_{1} e_{2}=\beta^{-1}$. Using this, we can rewrite (54) as

$$
0=E_{t}\left[\left(1-e_{1} L\right)\left(1-e_{2} L\right) \tilde{\pi}_{t+2}\right]
$$

Defining $z_{t} \equiv\left(1-e_{1} L\right) \tilde{\pi}_{t}$, this can be expressed as

$$
0=E_{t}\left[-e_{2}^{-1} L^{-1} L\left(1-e_{2} L\right) z_{t+2}\right]=E_{t}\left[\left(1-e_{2}^{-1} L^{-1}\right) z_{t+1}\right]
$$

or

$$
\begin{aligned}
E_{t} z_{t+1} & =E_{t}\left[e_{2}^{-1} z_{t+2}\right] \\
& =E_{t}\left[e_{2}^{-(T-t-1)} z_{T}\right],
\end{aligned}
$$

where the last equality is obtained after iterating forward. Using again the definition of $z_{t}$, we can then write

$$
E_{t}\left[\left(1-e_{1} L\right) \tilde{\pi}_{t+1}\right]=E_{t}\left[e_{2}^{-(T-t-1)}\left(\tilde{\pi}_{T}-e_{1} \tilde{\pi}_{T-1}\right)\right]
$$

or

$$
E_{t}\left[\tilde{\pi}_{t+1}-e_{1} \tilde{\pi}_{t}\right]=E_{t}\left[e_{2}^{-(T-t-1)}\left(\tilde{\pi}_{T}-e_{1} \tilde{\pi}_{T-1}\right)\right] .
$$

This expression can be iterated forward to yield

$$
\begin{aligned}
\tilde{\pi}_{t} & =E_{t}\left[-e_{1}^{-1} e_{2}^{-(T-t-1)}\left(\tilde{\pi}_{T}-e_{1} \tilde{\pi}_{T-1}\right)+e_{1}^{-1} \tilde{\pi}_{t+1}\right] \\
& =E_{t}\left[-e_{1}^{-(T-t)}\left(e_{1}^{T-t-1} e_{2}^{-(T-t-1)}+e_{1}^{T-t-2} e_{2}^{-(T-t-2)}+e_{1}^{T-t-3} e_{2}^{-(T-t-3)}+\ldots+e_{1}^{0} e_{2}^{0}\right)\left(\tilde{\pi}_{T}-e_{1} \tilde{\pi}_{T-1}\right)+e_{1}^{-(T-t)} \tilde{\pi}_{T}\right] \\
& =e_{1}^{-(T-t)} E_{t}\left[-\frac{1-\left(e_{1} e_{2}^{-1}\right)^{T-t}}{1-\left(e_{1} e_{2}^{-1}\right)}\left(\tilde{\pi}_{T}-e_{1} \tilde{\pi}_{T-1}\right)+\tilde{\pi}_{T}\right] .
\end{aligned}
$$

Note that (53) implies

$$
\begin{aligned}
& \pi_{T}=(1+\kappa \sigma) \bar{\pi}^{*}+\kappa \sigma \underline{r} \\
& \tilde{\pi}_{T}=\pi_{T}-\underline{\pi}=(1+\kappa \sigma)\left(\bar{\pi}^{*}+\underline{r}\right)
\end{aligned}
$$

and

$$
\begin{aligned}
& \pi_{T-1}=(1+\beta+\kappa \sigma) \pi_{T}-\beta \bar{\pi}^{*}+\kappa \sigma \underline{r}=\left((1+\kappa \sigma)^{2}+\beta \kappa \sigma\right) \bar{\pi}^{*}+(2+\beta+\kappa \sigma) \kappa \sigma \underline{r} \\
& \tilde{\pi}_{T-1}=\pi_{T-1}-\underline{\pi}=\left((1+\kappa \sigma)^{2}+\beta \kappa \sigma\right)\left(\bar{\pi}^{*}+\underline{r}\right)
\end{aligned}
$$

It follows, using $\beta e_{1} e_{2}=1$, that

$$
\begin{aligned}
\tilde{\pi}_{t} & =e_{1}^{-(T-t)}\left[-\frac{1-\left(e_{1} e_{2}^{-1}\right)^{T-t}}{1-\left(e_{1} e_{2}^{-1}\right)}\left((1+\kappa \sigma)-e_{1}\left((1+\kappa \sigma)^{2}+\beta \kappa \sigma\right)\right)+(1+\kappa \sigma)\right]\left(\bar{\pi}^{*}+\underline{r}\right) \\
& =\left[\frac{\left(\beta e_{1}\right)^{T-t}-\left(\beta e_{2}\right)^{T-t}}{1-\left(e_{1} e_{2}^{-1}\right)}\left((1+\kappa \sigma)-e_{1}\left((1+\kappa \sigma)^{2}+\beta \kappa \sigma\right)\right)+\left(\beta e_{2}\right)^{T-t}(1+\kappa \sigma)\right]\left(\bar{\pi}^{*}+\underline{r}\right)
\end{aligned}
$$


or

$$
\pi_{t}=\left\{\begin{array}{lc}
-\underline{r}+c_{1}\left(\beta e_{1}\right)^{T-t}+c_{2}\left(\beta e_{2}\right)^{T-t} & \text { for } t=0,1, \ldots T \\
\bar{\pi}^{*} & \text { for } t>T
\end{array}\right.
$$

where

$$
\begin{aligned}
c_{1} & =\left(1-e_{1} e_{2}^{-1}\right)^{-1}\left((1+\kappa \sigma)-e_{1}\left((1+\kappa \sigma)^{2}+\beta \kappa \sigma\right)\right)\left(\bar{\pi}^{*}+\underline{r}\right) \\
c_{2} & =\left((1+\kappa \sigma)-\frac{1}{1-e_{1} e_{2}^{-1}}\left((1+\kappa \sigma)-e_{1}\left((1+\kappa \sigma)^{2}+\beta \kappa \sigma\right)\right)\right)\left(\bar{\pi}^{*}+\underline{r}\right) \\
& =\left(1-e_{1} e_{2}^{-1}\right)^{-1}\left(\left(1+\kappa \sigma-e_{2}^{-1}\right)(1+\kappa \sigma)+\beta \kappa \sigma\right) e_{1}\left(\bar{\pi}^{*}+\underline{r}\right) .
\end{aligned}
$$

Given that $\underline{r}+\bar{\pi}^{*}<0$, we have $c_{2}<0$. In addition, $c_{1}+c_{2}=(1+\kappa \sigma)\left(\bar{\pi}^{*}+\underline{r}\right)<0$, so that in period $T$, $\pi_{T}=-\underline{r}+c_{1}+c_{2}<-\underline{r}$.

To solve for output, we iterate forward (52) to obtain

$$
\begin{aligned}
\hat{Y}_{t} & =E_{t} \sum_{j=1}^{T-t} \sigma\left(\pi_{t+j}+\underline{r}\right)+E_{t} \hat{Y}_{T}=\sum_{j=1}^{T-t} \sigma\left(c_{1}\left(\beta e_{1}\right)^{T-t-j}+c_{2}\left(\beta e_{2}\right)^{T-t-j}\right)+\bar{Y}+\sigma\left(\bar{\pi}^{*}+\underline{r}\right) \\
& =\bar{Y}+\sigma\left[\bar{\pi}^{*}+\underline{r}+c_{1} \frac{1-\left(\beta e_{1}\right)^{T-t}}{1-\beta e_{1}}+c_{2} \frac{1-\left(\beta e_{2}\right)^{T-t}}{1-\beta e_{2}}\right] \\
& =\bar{Y}+\sigma\left[\bar{\pi}^{*}+\underline{r}+\frac{c_{1}}{1-\beta e_{1}}+\frac{c_{2}}{1-\beta e_{2}}-c_{1} \frac{\left(\beta e_{1}\right)^{T-t}}{1-\beta e_{1}}-c_{2} \frac{\left(\beta e_{2}\right)^{T-t}}{1-\beta e_{2}}\right] \\
& =c_{0}-\sigma c_{1} \frac{\left(\beta e_{1}\right)^{T-t}}{1-\beta e_{1}}-\sigma c_{2} \frac{\left(\beta e_{2}\right)^{T-t}}{1-\beta e_{2}}
\end{aligned}
$$

where $c_{0} \equiv \bar{Y}+\sigma\left(\bar{\pi}^{*}+\underline{r}+\frac{c_{1}}{1-\beta e_{1}}+\frac{c_{2}}{1-\beta e_{2}}\right)$, and the second equality is obtained by noting that (52) implies $\hat{Y}_{T}=\bar{Y}+\sigma\left(\bar{\pi}^{*}+\underline{r}\right)$. 